\title{
Khellinoflavanone, a Semisynthetic Derivative of Khellin, Overcomes \\ Benzo[a]pyrene Toxicity in Human Normal and Cancer Cells that Express CYP1A1
}

\author{
Rajni Sharma, ${ }^{\dagger \neq}$ Ibidapo S. Williams, ${ }^{\| \# \perp}$ Linda Gatchie, ${ }^{\|}$Vinay R. Sonawane, ${ }^{\| \phi}$ Bhabatosh \\ Chaudhuri, ${ }^{\S \S *}$ Sandip B. Bharate ${ }^{\S \neq *}$
}

${ }^{\dagger}$ Natural Products Chemistry Division, CSIR-Indian Institute of Integrative Medicine, Canal Road, Jammu-180001, India

${ }^{\ddagger}$ Academy of Scientific \& Innovative Research, CSIR-Indian Institute of Integrative Medicine, Canal Road, Jammu-180001, India

${ }^{\S}$ Medicinal Chemistry Division, CSIR-Indian Institute of Integrative Medicine, Canal Road, Jammu180001, India

"CYP Design Ltd, Innovation Centre, 49 Oxford Street, Leicester, LE1 5XY, UK

Footnote:

${ }^{\perp}$ Current affiliation: Institute of Bio-sensing Technology, University of the West of England, Frenchay Campus, Filton Road, Bristol, BS34 8QZ, UK

ф Current affiliation: MedSphere India Private Limited, Goregaon (E), Mumbai-400065, India 


\section{ABSTRACT.}

Cytochrome P450 family 1 (CYP1) enzymes catalyze the metabolic activation of environmental procarcinogens such as benzo[a]pyrene, $\mathrm{B}[\mathrm{a}] \mathrm{P}$, into carcinogens, which initiate the process of carcinogenesis. Thus, stopping the metabolic activation of procarcinogens can possibly prevent the onset of cancer. Several natural products have been reported to show unique ability in inhibiting CYP1 enzymes. We found that khellin, a naturally occurring furanochromone from Ammi visnaga, inhibits CYP1A1 enzyme with an $\mathrm{IC}_{50}$ value of $4.02 \mu \mathrm{M}$ in CYP1A1-overexpressing human HEK293 suspension cells. To further explore this natural product for discovery of more potent and selective CYP1A1 inhibitors, two sets of semisynthetic derivatives were prepared. Treatment of khellin with alkali results in opening of a pyrone ring, yielding khellinone (2). Claisen-Schmidt condensation of khellinone (2) with various aldehydes in presence of potassium hydroxide, at room temperature, provide a series of furanochalcones 3a-v (khellinochalcones). Treatment of khellinone (2) with aryl aldehydes in presence of piperidine, under reflux, affords the flavanone series of compounds 4a-p (khellinoflavanones). The khellinoflavanone $4 \mathbf{l}$ potently inhibited CYP1A1 with IC50 value of $140 \mathrm{nM}$ in live cells, with 170-fold selectivity over CYP1B1 (IC50 for CYP1B1 = $23.8 \mu \mathrm{M}$ ). Compound $4 \mathbf{l}$ at $3 \mathrm{x}$ IC50 concentration for inhibition of CYP1A1, completely protected HEK293 cells from CYP1A1mediated B[a]P toxicity. Lung cancer cells, A549 (p53 ${ }^{+}$) and Calu-1 (p53-null), blocked in growth at the S-phase by $\mathrm{B}[\mathrm{a}] \mathrm{P}$ were restored into the cell cycle by compound $\mathbf{4 l}$. The results presented herein strongly indicate the potential of these khellin derivatives for further development as cancer chemopreventive agents.

KEYWORDS. Khellin; cytochrome P50 family 1 enzymes; CYP1A1; khellinone; flavanones; polycyclic aromatic hydrocarbons; cancer chemoprevention.

\section{INTRODUCTION}


Ammi visnaga (commonly known as 'Khella'; Family: Apiaceae) is a widespread flowering plant native to Europe, Asia, and North Africa, and has many therapeutic properties. ${ }^{1}$ The khella seeds are being used in traditional medicine for respiratory conditions including bronchitis, asthma, and cough. ${ }^{2}$ The seeds of this plant contain a $\gamma$-pyrone, furanochromone khellin as a major constituent, which is a vasodialator, and antispasmodic. ${ }^{1 \mathrm{a},} 3$ Inspired from the vasodialatory and antispasmodic property of khellin, Roger Altounyan, an Anglo-Armenian physician discovered cromoyln sodium, a chromone class of antiasthma drug. ${ }^{4}$ Khellin has also been extensively studied for its cardioactive properties, and is being used for angina pectoris therapy. ${ }^{5}$ Based on the cardioactive property of khellin, the Labaz company from Belgium, have discovered anti-arrhythmic drug amiodarone ${ }^{6}$ via medicinal chemistry on benzofuran scaffold. Other known pharmacological activities of khellin and its derivatives includes melanin-inhibition, ${ }^{1 \mathrm{~d}, 7}$ anti-inflammatory, ${ }^{8}$ anticancer, ${ }^{9}$ and cancer chemopreventive activity. ${ }^{10}$

Many natural compounds and their derivatives modulate the expression and activities of cytochrome P450 family 1 (CYP1) enzymes which are known to bioactivate procarcinogens. ${ }^{11}$ The cytochrome P450 proteins comprise a large family of enzymes that can catalyze the oxidative metabolism of a variety of xenobiotic chemicals including toxic compounds, drugs and chemical carcinogens as well as endogenous compounds. As a part of our continuing efforts to discover CYP1A1 inhibitors as cancer chemopreventive agents; $^{12}$ the furanochromone khellin (1) was identified as a selective CYP1A1 inhibitor $\left(\mathrm{CYP} 1 \mathrm{~A} 1 \mathrm{IC}_{50}=4.02 \mu \mathrm{M}, \mathrm{CYP} 1 \mathrm{~B} 1 \mathrm{IC}_{50}=34.6 \mu \mathrm{M}\right)$ via screening of institutional natural products repository (Figure 1). ${ }^{10}$

To further explore this natural product for fine-tuning its potency and selectivity profile, semisynthetic modifications were carried out. Literature reports indicated that semisynthetic modifications of khellin have been performed earlier, ${ }^{13}$ however, never been explored as cancer chemopreventive agents. Herein, we report synthesis of two series of khellin derivatives (khellinochalcones and khellinoflavanones) and their biological evaluation as CYP1A1 inhibitors and possible cancer chemopreventive agents. 
The in-vivo studies ${ }^{14}$ have demonstrated that CYP1A1 is involved in benzo[a]pyrene, B[a]P-induced carcinogenesis in mice. These studies have also shown that polycyclic aromatic hydrocarbon (PAH)treated mice overexpresses CYP1A1 in lungs and liver. Therefore, herein we also studied the ability of khellin derivatives to protect lung cancer cells and normal cells from B[a]P-induced toxicity.

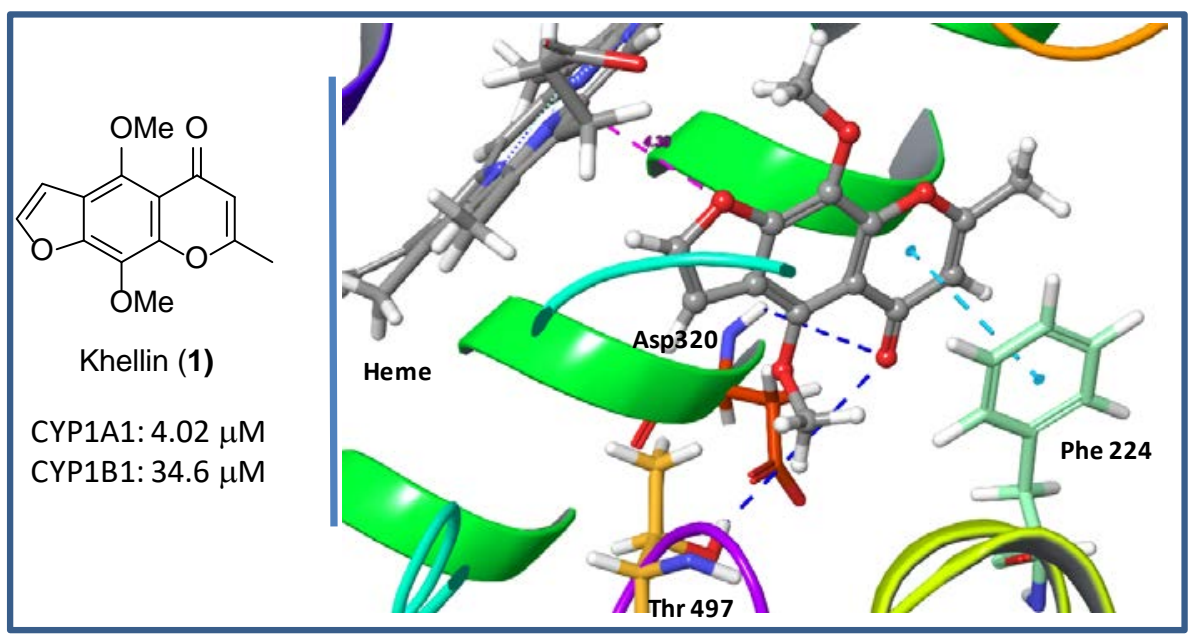

Figure 1. Structure of khellin (1), its CYP1A1, CYP1B1 inhibitory activity, and interactions with CYP1A1 active site. The H-bonding is shown by dark blue dotted lines, whereas $\pi$ - $\pi$ interactions by light-blue dotted lines.

\section{RESULTS AND DISCUSSION}

Synthesis of khellin derivatives. The khellin was identified as one of the 'hit' (active) from the natural products repository screening, ${ }^{10}$ in the search of new leads for cancer chemoprevention. However, the level of activity was in a micromolar range ( $\mathrm{IC}_{50}, 4.02 \mu \mathrm{M}$ ); therefore it was necessary to modify the structure of khellin to modulate its ability to inhibit CYP1A1. Based on the structural features of khellin, it was decided to convert khellin to khellinone followed by its condensation with aryl aldehydes. The presence of acetyl group in khellinone provides an easiest handle for chemical modifications. 
Khellin (1) was treated with potassium hydroxide in ethanol, resulting in formation of khellinone (2). ${ }^{15}$ Two series of compounds were prepared from khellinone $(2) ;{ }^{16}$ (a) chalcones by the ClaisenSchmidt condensation of khellinone with different substituted aryl aldehydes; and (b) furanoflavanones by treatment of khellinone with aryl aldehydes in presence of piperidine. The khellinone (2) was treated with aryl aldehydes under alkaline condition $(\mathrm{KOH})$, at room temperature, to obtain khellinochalcones 3a-v (Figure 2, path b). Interestingly, with slight modifications in the reaction conditions (base and reaction temperature), the another series of products were formed. Khellinone (2), when it was reacted with aryl aldehydes in ethanol with catalytic amounts of piperidine (instead of $\mathrm{KOH}$ ), under reflux conditions, it yielded furanoflavanones 4a-p (khellinoflavanones) (Figure 2 path c). Mechanistically, the flavanones were formed via cyclization of chalcones. All synthesized compounds were characterized based on their spectroscopic properties. The coupling constant of newly formed bond (C8-C9) in chalcones was found to be $16 \mathrm{~Hz}$, which confirmed the trans-orientation of the double bond. The ${ }^{1} \mathrm{H}$ NMR spectrum of flavanone $\mathbf{4 b}$ showed the signals of $\mathrm{H}-7$ at $\delta 5.45 \mathrm{ppm}$ and $\mathrm{H}-6$ at $\delta 3.04$ and 2.94 ppm confirming the formation of flavanone skeleton. Further, the ${ }^{13} \mathrm{C}$ NMR and DEPT135 spectrum confirmed the appearance of extra $-\mathrm{CH}_{2}$ at $\delta 45.0$ (C-6), and a - $\mathrm{CH}$ at $\delta 78.4$ (C-7). Interestingly, when khellinone (2) was treated with thiophene-2-carboxaldehyde, it underwent double aldehyde coupling, producing product $\mathbf{4 p}$. The ${ }^{1} \mathrm{H}$ NMR of $\mathbf{4 p}$ did not show presence of signal for $\mathrm{CH}_{2}$, however, the extra proton appeared at $\delta 7.13 \mathrm{ppm}$ indicating the presence of an additional olefinic proton.

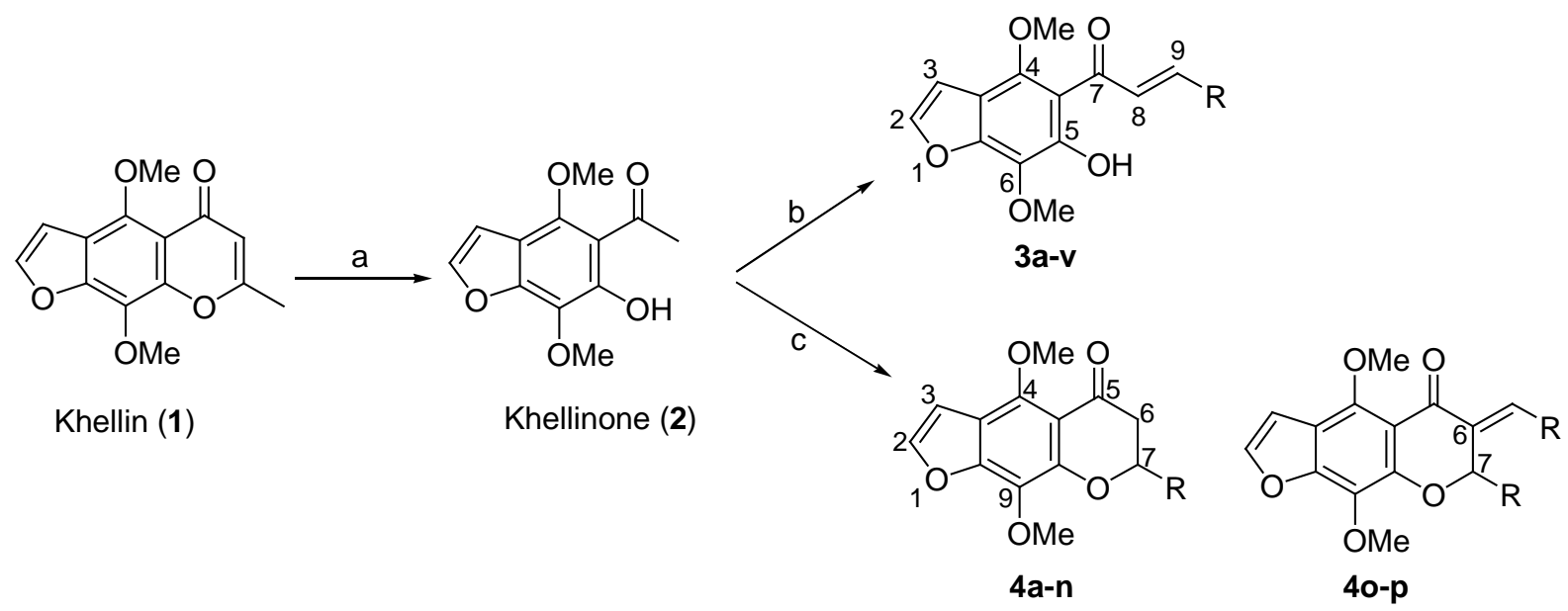


Figure 2. Synthesis of khellin derivatives 3a-v and 4a-p. Reagents and conditions (a) KOH (1M), ethanol, $80{ }^{\circ} \mathrm{C}, 12$ h, 61\%; (b) RCHO (R = aryl or heteroaryl), $\mathrm{KOH}(1 \mathrm{M})$, methanol, $0{ }^{\circ} \mathrm{C}$ to rt, 12-14 h, 40-85\%; (c) RCHO (R = aryl or heteroaryl), piperidine, ethanol, reflux at $70{ }^{\circ} \mathrm{C}, 12 \mathrm{~h}, 51-68 \%$. The 'R' groups of different derivatives 3a-v and 4a-p are shown in Table 1 and 3.

The pyridyl-furanochalcone derivative $3 g$ is a pan-inhibitor of CYP1 family enzymes. A series of furanochalcones 3a-v were tested for inhibition of CYP1A1 in yeast microsomes (Sacchrosomes ${ }^{\mathrm{TM}}$ ) and human live HEK293 cells, overexpressing CYP1A1, at $10 \mu \mathrm{M}$ (Table 1 ). ${ }^{10,12}$ The parent natural product khellin (1) showed 88\% and 81\% inhibition of CYP1A1, at $10 \mu \mathrm{M}$, in the two assays. Several chalcone derivatives showed higher inhibition of CYP1A1 in both assays. The furanochalcone 3a, bearing a simple phenyl ring (as R group) showed 92\% and 97\% inhibition of CYP1A1 in the microsomal and live cells assays. This result was expected, as chalcones have been reported to possess potent CYP1A1 inhibition activity. ${ }^{12 a}$ The most potent derivative among the series of chalcones was the compound $\mathbf{3 g}$, which bears a pyridin-3yl moiety as $\mathrm{R}$ group. This compound showed $97 \%$ and $99 \%$ inhibition of CYP1A1 in Sacchrosomes ${ }^{\mathrm{TM}}$ and in live human cells, respectively. The results of in-vitro CYP1A1 inhibition by furanochalcones in Sacchrosomes ${ }^{\mathrm{TM}}$ and HEK293 cells, at $10 \mu \mathrm{M}$ concentration, are shown in Table 1.

Table 1. In-vitro CYP1A1 inhibition by furanochalcones in Sacchrosomes ${ }^{\mathrm{TM}}$ and HEK293 cells, overexpressing CYP1A1, at $10 \mu \mathrm{M}$ concentration.

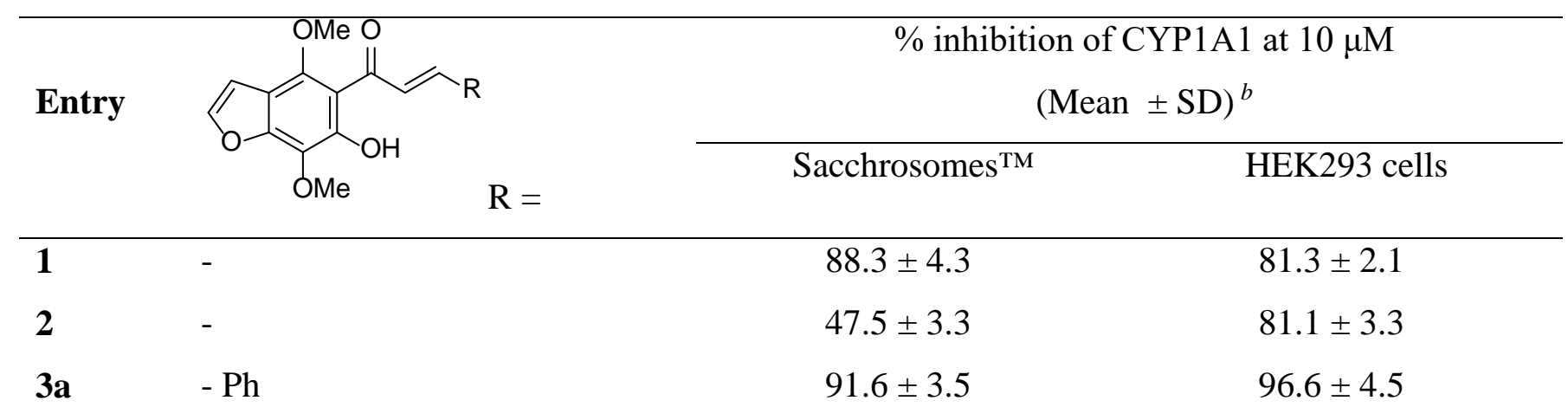




\begin{tabular}{llll} 
3b & - Ph (4-Br) & $26.8 \pm 2.3$ & $57.7 \pm 1.7$ \\
3c & - Ph (4-Cl) & $77.5 \pm 2.8$ & $85.7 \pm 2.1$ \\
3d & - Ph (3-Br,4-F) & $83.1 \pm 3.3$ & $82.3 \pm 2.1$ \\
3e & - Ph (4-F) & $92.4 \pm 3.6$ & $89.5 \pm 2.2$ \\
3f & - Ph (2,4-di-F) & $23.9 \pm 2.2$ & $85.4 \pm 2.8$ \\
3g & - pyridin-3-yl & $97.2 \pm 3.8$ & $99.2 \pm 3.3$ \\
3h & - Ph (4-OMe) & $28.9 \pm 2.3$ & $55.7 \pm 1.8$ \\
3i & - Ph (2,6-di-Cl) & $19.6 \pm 1.8$ & $58.8 \pm 1.8$ \\
3j & - Ph (2-OEt,5-Br) & $64.3 \pm 2.8$ & $31.7 \pm 1.5$ \\
3k & - Ph (2-Cl) & $23.6 \pm 2.1$ & $19.5 \pm 0.8$ \\
3l & - Ph (2,3-di-OMe) & $15.2 \pm 1.5$ & $21.6 \pm 0.9$ \\
3m & - Ph (2,4-di-OMe) & $10.2 \pm 1.1$ & $22.9 \pm 0.9$ \\
3n & - Ph (penta-F) & $21.9 \pm 1.8$ & $71.6 \pm 2.4$ \\
3o & - Ph (3-Br,4-OMe) & $37.0 \pm 2.4$ & $74.0 \pm 2.1$ \\
3p & - Ph (2,4,5-tri-OMe) & $92.2 \pm 3.5$ & $96.8 \pm 3.9$ \\
3q & - Ph (3,4-methylene-dioxy) & $48.4 \pm 2.7$ & $-1.1 \pm 2.1$ \\
3r & - thiophen-3-yl & $95.1 \pm 3.6$ & $79.4 \pm 2.8$ \\
3s & - Ph (2,4-di-Cl) & $80.2 \pm 3.3$ & $71.4 \pm 2.5$ \\
3t & - Ph (2-F) & $49.7 \pm 2.6$ & $-2.1 \pm 2.1$ \\
3u & - naphthalen-2-yl & $41.1 \pm 2.6$ & $0.2 \pm 2.6$ \\
3v & - anthracen-2-yl & $43.4 \pm 2.6$ & $-1.1 \pm 3.5$ \\
ANF & - & $97.1 \pm 2.6$ & $30.2 \pm 10.8$ \\
\hline
\end{tabular}

${ }^{a}$ ANF ( $\alpha$-naphthoflavone) was used as a positive control. ${ }^{b}$ The percentage inhibition values represent mean and standard deviations ( \pm SD) of results obtained from two independent experiments.

The IC 50 values were then determined for selected furanochalcones in a panel of enzymes belonging to cytochrome P450 sub-families 1, 2, and 3. The results are shown in Table 2. Chalcones 3e (bearing 4fluoro-phenyl as R group) and 3p (bearing 2,4,5-tri-OMe phenyl as R group) displayed highly selective inhibition of CYP1A1 with IC 50 value of 0.32 and $0.30 \mu \mathrm{M}$, respectively. These two compounds do not inhibit the other two enzymes from CYP1 sub-family, i.e. CYP1A2 or CYP1B1 (IC50 $>10 \mu \mathrm{M}$ ). Further, they do not inhibit the CYP2 and CYP3 sub-family of enzymes commonly involved in drug 
metabolism (Table 2). The pyridyl furanochalcone $\mathbf{3 g}$ showed inhibition of Sacchrosomes ${ }^{\mathrm{TM}}$ containing CYP1A1, CYP1A2 and CYP1B1 with $\mathrm{IC}_{50}$ values of $0.34,0.17$ and $0.66 \mu \mathrm{M}$, respectively; whereas no inhibition of CYP2 and CYP3 sub-family of enzymes was observed (IC $50>20 \mu \mathrm{M})$. The compound $\mathbf{3 g}$ showed $\mathrm{IC}_{50}$ values of 0.47 and $0.27 \mu \mathrm{M}$ for CYP1A1 and CYP1B1 inhibition in live human cell assays.

Table 2. $\mathrm{IC}_{50}$ values of selected furanochalcones in a panel of CYP enzymes in Sacchrosomes ${ }^{\mathrm{TM}}$

\begin{tabular}{|c|c|c|c|c|}
\hline \multirow[b]{2}{*}{ Entry } & \multicolumn{4}{|c|}{$\mathrm{IC}_{50}$ in $\mu \mathrm{M}$ (Sacchrosomes $\left.^{\mathrm{TM}}\right)^{a}$} \\
\hline & 1A1 & 1A2 & 1B1 & $\begin{array}{l}\text { 2A6, 2B6, 2C8, 2C9, 2C18, 2C19, } \\
\text { 2D6, 2E1, 3A4 }\end{array}$ \\
\hline $3 \mathbf{e}$ & $0.32 \pm 0.04$ & $>10$ & $>10$ & $>20$ \\
\hline $3 \mathbf{g}$ & $0.34 \pm 0.03$ & $0.17 \pm 0.02$ & $0.66 \pm 0.04$ & $>20$ \\
\hline $3 p$ & $0.30 \pm 0.05$ & $>20$ & $>20$ & $>20$ \\
\hline $3 \mathbf{r}$ & $0.28 \pm 0.03$ & $4.2 \pm 0.8$ & $0.78 \pm 0.04$ & $>20$ \\
\hline
\end{tabular}

${ }^{a}$ The $\mathrm{IC}_{50}$ values represent average ( \pm standard deviations) of results obtained from three independent experiments.

The in-silico docking studies have shown that the chalcone $\mathbf{3 g}$ displays H-bonding and $\pi-\pi$ interactions with the active site residues of CYP1A1. The carbonyl oxygen of the chalcone 3g showed three-way H-bonding interactions with Asp 320, Thr 497 and Asn 222 residues, whereas the pyridine ring displays $\pi-\pi$ stacking with Phe 224 and Phe 258 residues. The furan ring of the chalcone $3 \mathbf{g}$ has been oriented towards the heme of the CYP1A1 enzyme (Figure 3), as it was observed in case of khellin (Figure 1). 


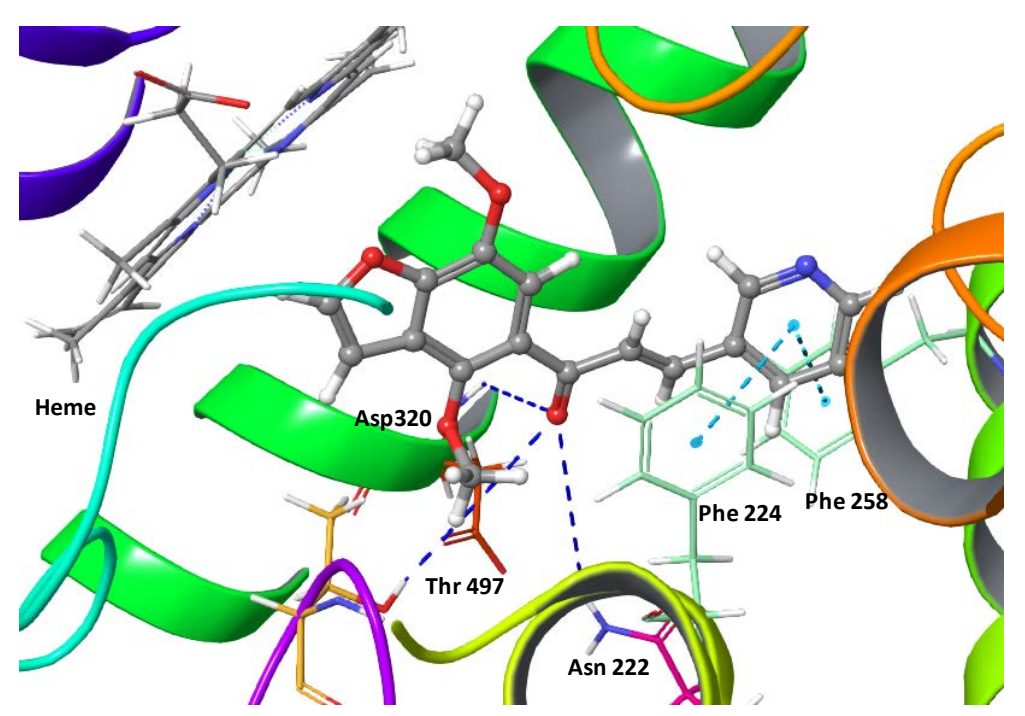

Figure 3. The interactions of chalcone 3g with CYP1A1 (PDB ID: 4I8V) active site. The H-bonding is shown by dark blue dotted lines, whereas $\pi$ - $\pi$ interactions by light-blue dotted lines.

The furanoflavanone derivative 41 is a potent and selective inhibitor of CYP1A1 enzyme. A series of furanoflavanones 4a-p were tested for inhibition of CYP1A1 enzyme borne on yeast microsomes (Sacchrosomes ${ }^{\mathrm{TM}}$ ) and CYP1A1-overexpressing HEK293 cells, at $10 \mu \mathrm{M}$ concentration (Table 3). Several flavanone derivatives showed $>85 \%$ inhibition of CYP1A1 enzyme in Sacchrosomes $^{\mathrm{TM}}$ as well as in live human cells overexpressing CYP1A1. The compound 4a, bearing a simple phenyl as R group, has shown $97 \%$ and $87 \%$ inhibition of CYP1A1 in Sacchrosomes ${ }^{\mathrm{TM}}$ and in live human cells, respectively. The 3-pyridyl substituted flavanone (compound 4e) also showed potent inhibition of CYP1A1 enzyme in both assay systems.

Table 3. In-vitro CYP1A1 inhibition by furanoflavanones in Sacchrosomes ${ }^{\mathrm{TM}}$ and HEK293 suspension cells, overexpressing CYP1A1, at $10 \mu \mathrm{M}$ concentrations. ${ }^{a}$

$\underset{1}{\mathrm{OMe}} \mathrm{O}_{11} \quad \mathrm{OMe}_{11}^{\mathrm{O}} \quad \%$ inhibition of CYP1A1 at $10 \mu \mathrm{M}$




\begin{tabular}{|c|c|c|c|}
\hline \multirow{2}{*}{ Entry } & \multirow[t]{2}{*}{$\mathrm{R}=$} & \multicolumn{2}{|c|}{$(\text { Mean } \pm \mathrm{SD})^{b}$} \\
\hline & & Sacchrosomes ${ }^{\mathrm{TM}}$ & HEK293 cells \\
\hline $4 \mathbf{a}$ & $-\mathrm{Ph}$ & $97.0 \pm 2.4$ & $87.4 \pm 3.4$ \\
\hline $4 b$ & - $\mathrm{Ph}(2-\mathrm{Br})$ & $97.0 \pm 3.1$ & $98.5 \pm 2.1$ \\
\hline $4 c$ & - Ph (3,4-methylene-dioxy) & $78.1 \pm 1.4$ & $74.1 \pm 3.4$ \\
\hline 4d & - Ph (5-Br, 2-OMe) & $87.8 \pm 3.9$ & $72.6 \pm 5.2$ \\
\hline $4 e$ & - pyridin-3-yl & $96.8 \pm 3.2$ & $87.0 \pm 2.5$ \\
\hline $4 f$ & - thiophen-3-yl & $79.9 \pm 0.4$ & $44.3 \pm 4.7$ \\
\hline $4 g$ & $-\mathrm{Ph}\left(4-\mathrm{NO}_{2}\right)$ & $48.1 \pm 2.9$ & $-1.5 \pm 2.9$ \\
\hline $4 h$ & - Ph (2-Cl) & $87.1 \pm 0.9$ & $56.9 \pm 6.2$ \\
\hline $4 i$ & - Ph (2,4-di-Cl) & $94.3 \pm 2.3$ & $72.6 \pm 3.5$ \\
\hline $4 \mathbf{j}$ & - naphthalen-2-yl & $71.7 \pm 4.2$ & $72.2 \pm 2.7$ \\
\hline $4 \mathbf{k}$ & - Ph (2,6-di-Cl) & $75.6 \pm 1.2$ & $54.1 \pm 2.4$ \\
\hline 41 & - Ph (3-Br,4-F) & $96.2 \pm 1.8$ & $89.4 \pm 1.3$ \\
\hline $4 m$ & $-\mathrm{Ph}(2-\mathrm{F})$ & $97.1 \pm 1.4$ & $77.8 \pm 3.1$ \\
\hline $4 n$ & $-\mathrm{Ph}(4-\mathrm{F})$ & $94.2 \pm 1.5$ & $79.5 \pm 2.3$ \\
\hline 40 & $-\mathrm{Ph}$ & $60.7 \pm 0.5$ & $0.4 \pm 2.4$ \\
\hline $4 p$ & -thiophene-3-yl & $95.8 \pm 4.2$ & $68.5 \pm 2.9$ \\
\hline ANF & - & $97.1 \pm 2.6$ & $30.2 \pm 10.8$ \\
\hline
\end{tabular}

${ }^{a}$ The IC 50 values represent average ( \pm standard deviation) of results obtained from three independent experiments.

Next, the $\mathrm{IC}_{50}$ values were determined for three best flavanones, in live human cell assays expressing specific CYP enzymes. Compound 4a was found to be a dual inhibitor of CYP1A1 and CYP1B1 with slight selectivity towards CYP1B1. However, the compounds $\mathbf{4 e}$ and $\mathbf{4 l}$ were selective CYP1A1 inhibitors, showing 9.2 and 170-fold selectivity over CYP1B1 (Table 4). These compounds does not inhibit CYP1A2, CYP2D6 and CYP3A4 ( $\left.\mathrm{IC}_{50}>10 \mu \mathrm{M}\right)$. The excellent selectivity profile of flavanone $4 \mathbf{l}$ against CYP2D6, CYP3A4 and also within the CYP family 1 enzymes, indicated its potential for further exploration. 
Table 4. IC $_{50}$ values of selected furanochalcones for CYP1A1, CYP1A2, CYP1B1, CYP2D6 and CYP3A4 inhibition in live human HEK293 cells

\begin{tabular}{lccccc}
\hline \multirow{2}{*}{ Entry } & \multicolumn{5}{c}{${\text { IC50 in } \boldsymbol{\mu M} \text { (live cells) }^{a}}$} \\
\cline { 2 - 6 } & CYP1A1 & CYP1A2 & CYP1B1 & CYP2D6 & CYP3A4 \\
\hline $\mathbf{4 a}$ & $1.84 \pm 0.14$ & $>10$ & $0.566 \pm 0.08$ & $>10$ & $>10$ \\
$\mathbf{4 e}$ & $0.45 \pm 0.06$ & $>10$ & $4.16 \pm 0.25$ & $>10$ & $>10$ \\
$\mathbf{4 l}$ & $0.14 \pm 0.04$ & $>10$ & $23.8 \pm 1.2$ & $>10$ & $>10$
\end{tabular}

${ }^{a}$ The $\mathrm{IC}_{50}$ values represent average ( \pm standard deviation) of results obtained from three independent experiments.

The in-silico docking studies of flavanone $\mathbf{4 l}$ with CYP1A1 have shown that it display interactions similar to chalcone 3g; however, the molecule was found to be flipped by $180^{\circ}$ inside the active site. The furan ring of chalcone $\mathbf{3 g}$ was oriented towards the heme, whereas it is away from heme in case of flavanone 4l. However, despite this flip, the three-way H-bonding interaction of carbonyl oxygen with Asp 320, Thr 497 and Asn 222 residues was common in both the inhibitors. The flavanone $4 \mathbf{l}$ also showed two $\pi-\pi$ stacking interactions with Phe 224 and Phe 258 residues. The interactions of $4 \mathbf{l}$ with CYP1A1 are shown in Figure 4. 


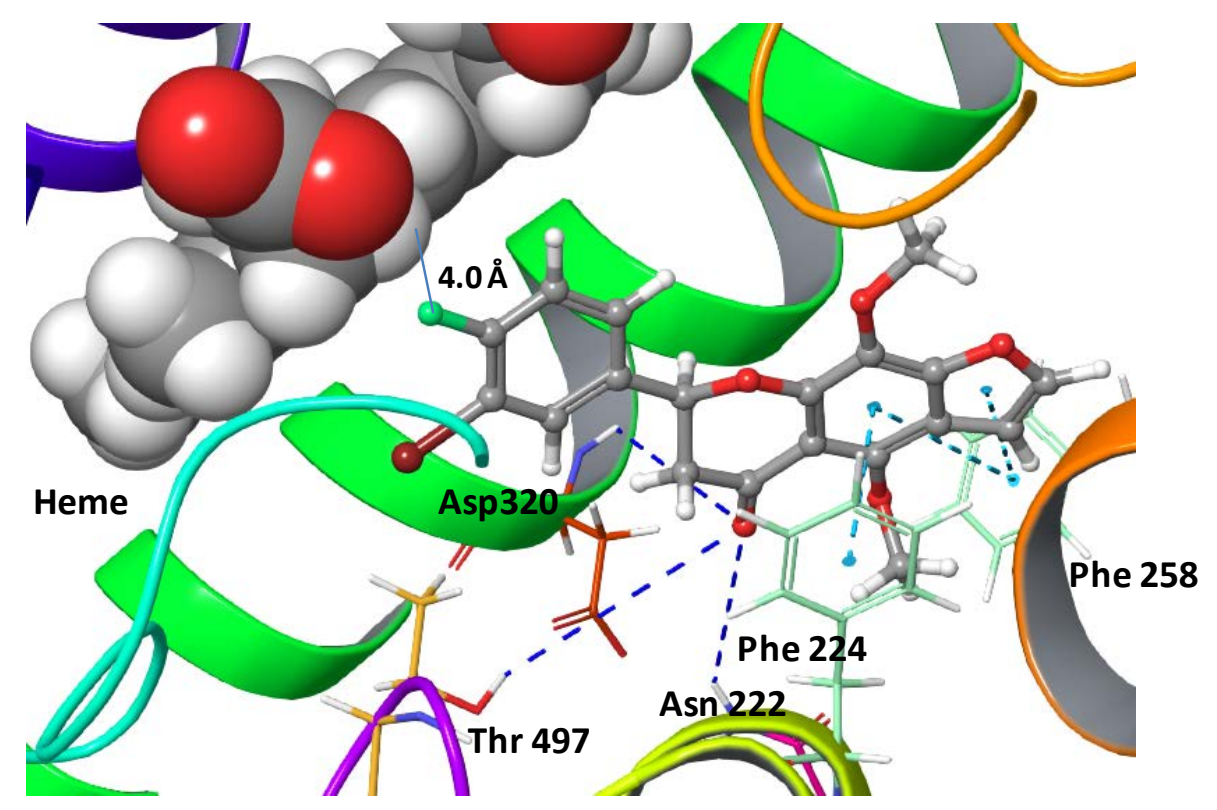

Figure 4. The interactions of flavanone $4 \mathbf{l}$ with CYP1A1 (PDB ID: 4I8V) active site.

On comparison of the CYP1A1 and CYP1B1 inhibition data of naphthyl pyridochalcones published earlier by us, ${ }^{17}$ with the data of khellinochalcones and khellinoflavanones, an interesting structureactivity relationship was observed. The 3-pyridyl naphthyl chalcone ${ }^{17}$ (structure shown in Figure 5) has been reported as potent and selective CYP1B1 inhibitor with an $\mathrm{IC}_{50}$ value of $4 \mathrm{nM}$, showing selectivity over CYP1A1. The replacement of naphthyl ring with 4,7-dimethoxybenzofuran has resulted in improved CYP1A1 inhibitory activity (Figure 5). The 4,7-dimethoxybenzofuran ring bearing 3-pyridyl chalcone $3 g$ showed CYP1A1 inhibition with $\mathrm{IC}_{50}$ value of $470 \mathrm{nM}$. Compound 3g inhibited CYP1B1 in live human cell assay with an $\mathrm{IC}_{50}$ of $270 \mathrm{nM}$, indicating slight selectivity towards CYP1B1. Furthermore, when the chalcone skeleton was cyclized in the form of a flavanone (3g versus 4e), there was a huge gain in CYP1A1 selectivity. This indicated that flavanones have better selectivity towards CYP1A1, compared with chalcones. Interestingly, when the pyridyl ring of flavanone 4e was replaced with substituted phenyl, the potent and selective CYP1A1 inhibitor $\mathbf{4 l}$ was identified. The compound $\mathbf{4 l}$ bearing 3-bromo-4-fluorophenyl ring instead of 3-pyridyl (of 4e), inhibited CYP1A1 with IC 50 value of 
$140 \mathrm{nM}$, and 170-fold selectivity over CYP1B1 (IC50 for CYP1B1 = $23.8 \mu \mathrm{M}$ ). The CYP1A1 and CYP1B1 activities of chalcones and flavanones, indicating selectivity within these two enzymes, is depicted in Figure 5.<smiles>COc1ccc2cc(C(=O)/C=C/c3cccnc3)ccc2c1</smiles>

Compound 6j from ref. 17

CYP1A1: $795 \mathrm{nM}$ CYP1B1: 4 nM

CYP1B1 selective inhibitor<smiles>COc1c2c(c(OC)c3occc13)OC(c1ccc(F)c(Br)c1)CC2=O</smiles>

4I

CYP1A1: $140 \mathrm{nM}$

CYP1B1: $23800 \mathrm{nM}$

CYP1A1 selective inhibitor
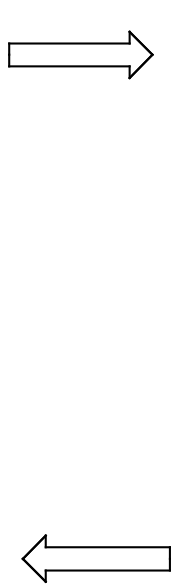<smiles>COc1c2c(c(OC)c3occc13)OC(c1cccnc1)CC2=O</smiles>

$4 e$

CYP1A1: $450 \mathrm{nM}$ CYP1B1: $4160 \mathrm{nM}$

Figure 5. Structure-activity relationship between chalcones and flavanones.

\section{Furanochalcone $3 \mathrm{~g}$ and furanoflavanone $4 \mathrm{l}$ protect CYP1A1-overexpressing normal human} cells from CYP1A1 mediated B[a]P toxicity. CYP1A1 is an aryl hydroxylase which provides metabolic activation of polycyclic aromatic hydrocarbons (PAHs) and polyhalogenated aromatic hydrocarbons (PHAHs). It is mainly expressed in the extrahepatic tissue like lung, placenta, skin etc. ${ }^{18}$ The CYP1A1 enzyme is known to metabolically activate benzo[a]pyrene to its carcinogenic form. ${ }^{18-19}$ Therefore, to investigate the potential of identified CYP1A1 inhibitors to stop the process of metabolic activation, thereby protecting normal cells from carcinogenesis, the B[a]P toxicity in normal HEK293 cells and HEK293 cells, ${ }^{20}$ overexpressing CYP1A1, was studied. ${ }^{10,}{ }^{12}$ The $\mathrm{EC}_{50}$ of $\mathrm{B}[\mathrm{a}] \mathrm{P}$ in normal HEK293 cells was found to be $14 \mu \mathrm{M}$, whereas in CYP1A1 overexpressing cells, it was highly toxic 
(EC50 $1.4 \mu \mathrm{M}$ ), indicating that CYP1A1 metabolizes $\mathrm{B}[\mathrm{a}] \mathrm{P}$ to its toxic form. The co-treatment of these CYP1A1 overexpressing cells with CYP1A1 inhibitors $\mathbf{3 g}$ or $\mathbf{4 l}$, at their $3 \mathrm{x} \mathrm{IC}_{50}$ concentrations, resulted in nullifying of $\mathrm{B}[\mathrm{a}] \mathrm{P}$ toxicity. The cells were completely protected from $\mathrm{B}[\mathrm{a}] \mathrm{P}$ toxicity as indicated by restoration of the B[a]P EC50 to normal (i.e. $14 \mu \mathrm{M}$ ) (Figure 6).

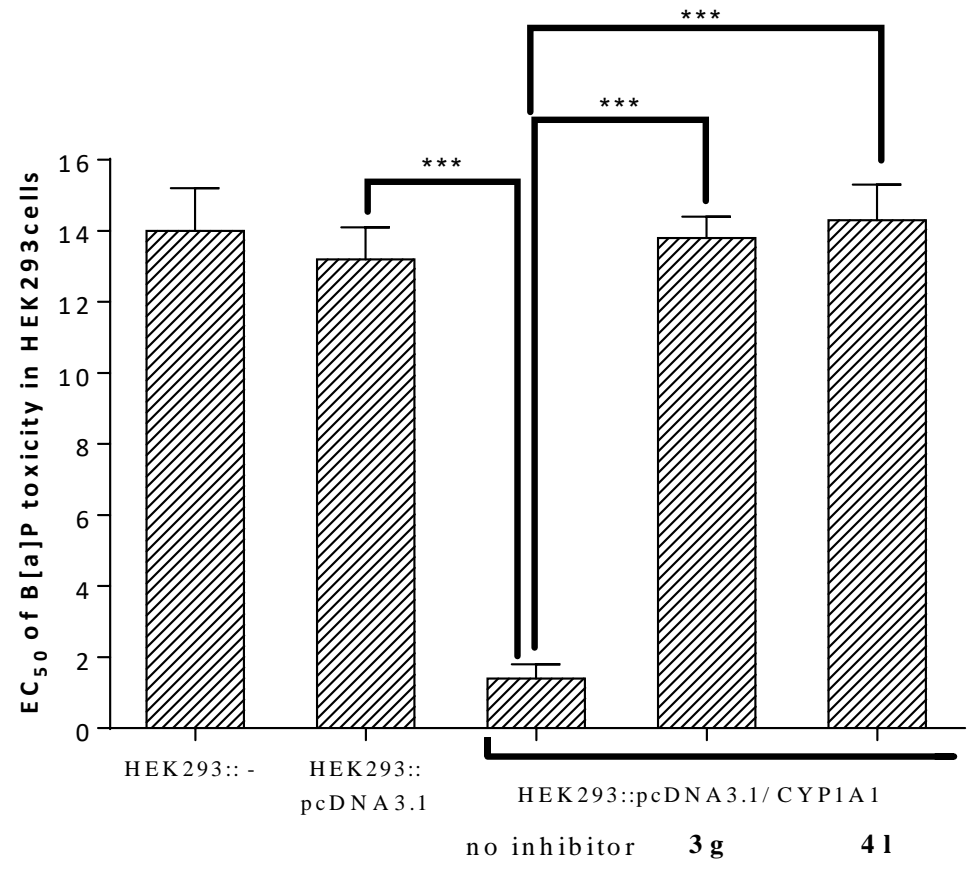

Figure 6. Protection of CYP1A1-overexpressing HEK293 cells from B[a]P toxicity. B[a]P was used in the concentration range of $0.05 \mu \mathrm{M}-100 \mu \mathrm{M}$, in the presence of $3 \times \mathrm{IC}_{50}$ values of the compounds $3 g$ or 41 (as determined in the human cell assay where cells expressed CYP1A1 when they were grown in suspension). The cell viability was determined by MTT assay. The cell viability determination using sulforhodamine B (SRB) assay ${ }^{21}$ provided similar results to the MTT assay. EC 50 values are presented in $\mu \mathrm{M}$ concentrations, and represents the average of three independent experiments (***P $<0.001$ ).

\section{Furanoflavanone 41 prevents B[a]P-mediated S-phase block in human lung cancer cells, A549}

and Calu-1. The human lung cancer cell lines A549 22 and Calu- ${ }^{23}$ are known to express CYP1A1 enzyme. Furthermore, the in vivo studies in mice model ${ }^{14}$ have shown that PAH-treated mice overexpress CYP1A1 in lung tissues; therefore lung cancer cell lines were chosen for this study. The effect of CYP1A1 inhibitors on the cell cycle, in the presence of $\mathrm{B}[a] \mathrm{P}$, was investigated by first pretreating A549 (ATCC CCL-185) and Calu-1 (ATCC HTB-54) lung cancer cells (which express 
CYP1A1; Figure 7A) with the most potent CYP1A1 inhibitor, compound $\mathbf{4 l}$, and then exposing cells to $\mathrm{B}[a] \mathrm{P}$. The endogenous CYP1A1 is expected to metabolize $\mathrm{B}[a] \mathrm{P}$ to its diol-epoxide (BPDE) and corresponding O-quinone, products which are known to intercalate DNA. DNA-intercalation should block cells primarily at the S phase of the cell division cycle. It has been suggested that DNA-adduct formation cause DNA mutations that lead to the onset of cancer. ${ }^{24}$

Upon exposure of asynchronous A549 and Calu-1 lung cancer cells (Figures 7B and E) to a $15 \mu \mathrm{M}$ concentration of $\mathrm{B}[a] \mathrm{P}$ for $3 \mathrm{~h}$ and then releasing cells into fresh medium for $57 \mathrm{~h}$, the cells did face a block at the S phase of the cell cycle (Figures 7C and F). This would be expected if the CYP1A1 protein (Figure 7A), expressed within A549 and Calu-1cells, were to be a functional enzyme. However, when cells were at first pre-incubated for $20 \mathrm{~h}$ with the CYP1A1-specific inhibitor, compounds $\mathbf{4 l}$, at its 3x $\mathrm{IC}_{50}$ concentrations, and then treated with a $15 \mu \mathrm{M}$ concentration of $\mathrm{B}[\mathrm{a}] \mathrm{P}$ for $3 \mathrm{~h}$, followed by release into fresh medium, the cells were preponderantly released from the block (Figures 7D and G). This would imply that CYP1A1-mediated metabolism of $\mathrm{B}[a] \mathrm{P}$ in A549 and Calu-1 cells has been prevented by compound $\mathbf{4 l}$, demonstrating the efficacy of the compound in preventing $\mathrm{B}[\mathrm{a}] \mathrm{P}$-mediated toxicity in lung cancer cells. 


\section{(A) $\quad 1 \quad 2 \quad 3 \quad 3 \quad 4 \quad 5$}

\section{CYP1A1}

$\beta$-Actin
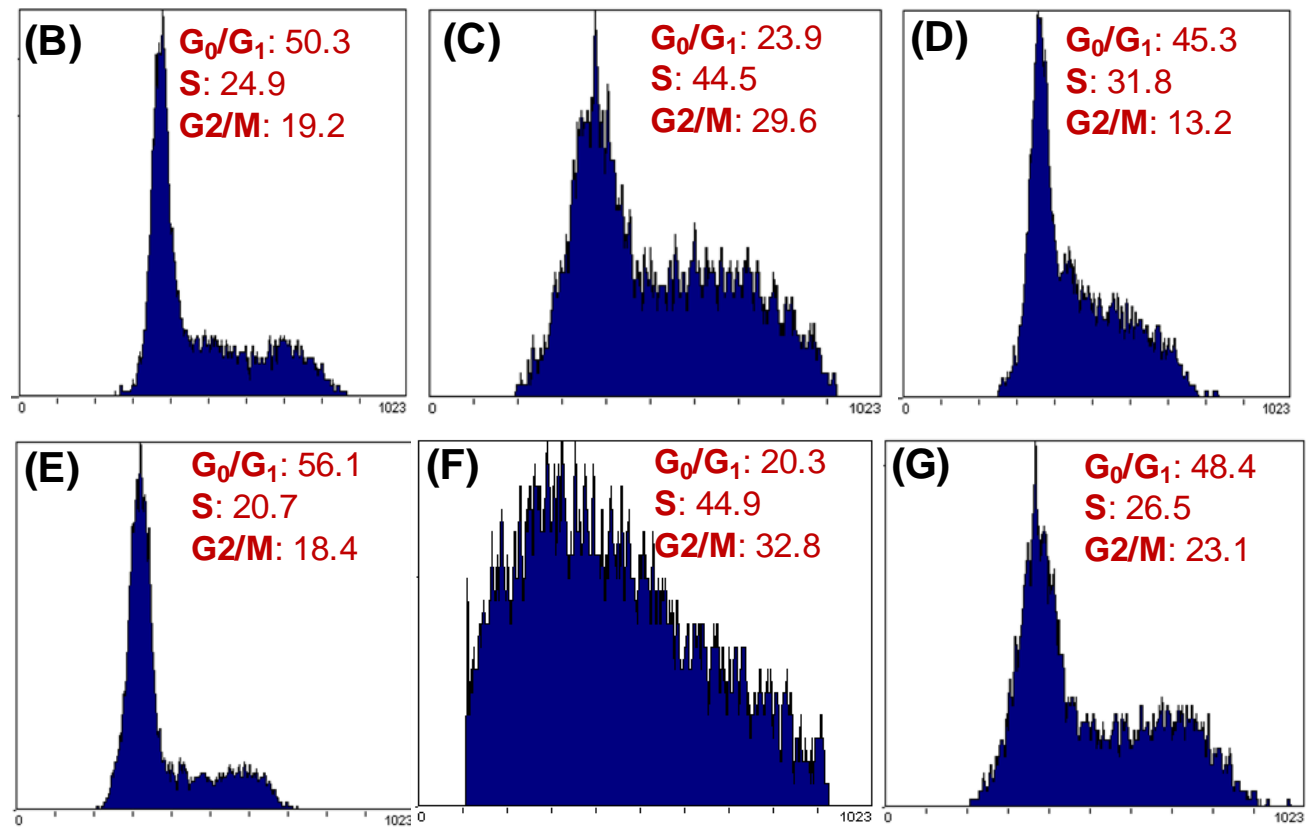

Figure 7. Western blot and FACS analyses of A549 and Calu-1 lung cancer cells. (A)Expression of CYP1A1, in $5 \mu \mathrm{g}$ of total protein, isolated from A549 (lane 1), Calu-1cells (lane 3), normal HEK293 cells (lane 2; as negative control) and normal WI-38 cells (lane 4; as negative control) was confirmed by Western blotting; CYP1A1-bearing Sacchrosomes ${ }^{\mathrm{TM}}$ (1pmol) were taken as positive control (lane 5). The CYP1A1-specificantibody, used for confirming CYP1A1 expression in the upper panel, was from ProteinTech(\#13241-1-A); beta-actin was used as a loading control and the blot (lower panel) was probed with an antibody from Sigma (\# A2228). (B) and (E): FACS analyses of untreated A549 (B) and Calu-1 cells (E)growing asynchronously. FACS analyses of A549 cells, treated with B[a]P, in the absence of compound $4 \mathbf{l}(\mathrm{C})$ and in the presence of compound $\mathbf{4 l}$ (D). FACS analyses of Calu-1 cells, treated with $\mathrm{B}[a] \mathrm{P}$, in the absence of compound $4 \mathrm{l}(\mathrm{F})$ and in the presence of compound $4 \mathbf{l}(\mathrm{G})$. The percentage of cells in $G_{0} / G_{1}, S$ and $G_{2} / M$ phases of the cell division cycle is shown in the upper righthand corner of each of the FACS analyses. 


\section{CONCLUSION}

In summary, we have identified that the semisynthetic modifications on the natural product khellin has resulted in significant improvement in its CYP1A1 inhibitory activity. The furanochalcone derivative $\mathbf{3 g}$ and furanoflavanone derivative $\mathbf{4 l}$ displayed potent inhibition of CYP1A1 with IC $_{50}$ values of 470 and $140 \mathrm{nM}$, respectively. These inhibitors completely protected normal human cells from the toxicity of procarcinogen $\mathrm{B}[\mathrm{a}] \mathrm{P}$ via inhibition of its metabolic activation. Moreover, compound $\mathbf{4 l}$ also prevented manifestation of B[a]P-mediated S-phase block (i.e. DNA-intercalation that occurs at S-phase upon $\mathrm{B}[\mathrm{a}] \mathrm{P}$ treatment) in lung cancer cells. Thus, the leads identified herein have potential for further investigation as cancer chemopreventive agents.

\section{EXPERIMENTAL SECTION}

General. The commercially available khellin was purchased from Sigma Aldrich (CAS: 82-02-0, Lot $\neq$ BCBJ3836U). NMR spectra were recorded on Bruker-Avance DPX FT-NMR 500 and $400 \mathrm{MHz}$ instruments. Chemical shift values for protons/ carbons are reported in parts per million (ppm) downfield from tetramethylsilane. ESI-MS and HRMS spectra were recorded on Agilent 1100 LC-QTOF and HRMS-6540-UHD machines. IR spectra were recorded on Perkin-Elmer IR spectrophotometer. Melting points were recorded on digital melting point apparatus.

The purity of all derivatives was determined using HPLC analysis. The sample solution of compounds of concentration $0.5 \mathrm{mg} / \mathrm{ml}$ prepared in acetonitrile and filtered through $0.45 \mu$ membrane filter injected $(15 \mu \mathrm{l})$ in the HPLC system (Waters) using Chromolith C-18e column (Merck, 50 X $4.6 \mathrm{~mm}$ ). The binary mobile phase consisted of A (water) and B (acetonitrile) was used. The isocratic elution was used as follows: 80 min run time with $90 \%$ acetonitrile and $10 \%$ water. The flow rate was $1 \mathrm{~mL} /$ minutes and the results were analyzed using UV-PDA detector.

Synthesis of khellinone (2). The mixture of khellin (900 mg, $3.46 \mathrm{mmol}$ ) and potassium hydroxide (1 M) in ethanol was refluxed at $90{ }^{\circ} \mathrm{C}$ over a period of $12-14 \mathrm{~h}$. After completion of the reaction, ethanol 
was evaporated over vacuo rotavapor and the obtained residue was partitioned in between dichloromethane and water. The dichloromethane layer was washed with brine solution and was dried over anhydrous sodium sulphate. The solvent was evaporated over vacuo rotavapor and the obtained crude product was purified using silica gel column chromatography (5-10\% ethyl acetate in hexane) to get khellinone (2) as a yellow powder. The purchased khellin along with khellinone were characterized using spectral analysis.

Khellin (1): white crystals; HPLC: $t_{\mathrm{R}}=4.6 \mathrm{~min}$ (99\% purity); m.p. $155-156{ }^{\circ} \mathrm{C}$; IR $\left(\mathrm{CHCl}_{3}\right): v_{\max }$ 3436, 3160, 3137, 2989, 2931, 2960, 2830, 1619, 1586, 1471, 1444, 1424, 1364, 1380, 1300, $1265 \mathrm{~cm}^{-1}$; ${ }^{1} \mathrm{H}$ NMR (400 MHz, $\left.\mathrm{CDCl}_{3}\right): \delta(\mathrm{ppm}) 7.51$ (d, $\left.J=2.2 \mathrm{~Hz}, 1 \mathrm{H}, \mathrm{CH}\right), 6.91(\mathrm{~d}, J=4.0 \mathrm{~Hz}, 1 \mathrm{H}, \mathrm{CH}), 4.15$ (s, 3H, OMe), 4.05 (s, 3H, OMe), 2.73 (s, 3H, Me); ${ }^{13} \mathrm{C}$ NMR (100 MHz, $\left.\mathrm{CDCl}_{3}\right): \delta$ (ppm) $206.2(\mathrm{C}=\mathrm{O})$, 153.5 (C-7a), 152.3 (C-6), 151.6 (C-3), 143.8 (OㅡH=CH), 128.8 (C-7), 110.8 (C-3a), 110.5 (C-5), 106.7 $(\mathrm{OCH}=\underline{\mathrm{CH}}), 61.0$ (OMe), 60.9 (OMe), 33.2 (Me); HR-ESIMS: m/z $237.0759[\mathrm{M}+\mathrm{H}]^{+}$calcd for $\mathrm{C}_{12} \mathrm{H}_{12} \mathrm{O}_{5}+\mathrm{H}^{+}(237.0757) .{ }^{25}$

1-(6-Hydroxy-4,7-dimethoxy-benzofuran-5-yl) (2): yellow crystals; HPLC: $t_{\mathrm{R}}=4.6$ min (99\% purity); yield: 61\%; m.p. $169-170{ }^{\circ} \mathrm{C}$; IR $\left(\mathrm{CHCl}_{3}\right): v_{\max } 3436,3160,3137,2989,2931,2960,2830,1619,1586$, 1471, 1444, 1424, 1364, 1380, 1300, $1265 \mathrm{~cm}^{-1} ;{ }^{1} \mathrm{H}$ NMR (400 MHz, $\left.\mathrm{CDCl}_{3}\right): \delta(\mathrm{ppm}) 7.51$ (d, $J=2.2$ Hz, 1H, CH), 6.91 (d, J = 4.0 Hz, 1H, CH), 4.15 (s, 3H, OMe), 4.05 (s, 3H, OMe), 2.73 (s, 3H, Me); ${ }^{13} \mathrm{C}$ NMR (100 MHz, $\left.\mathrm{CDCl}_{3}\right): \delta(\mathrm{ppm}) 206.2$ (C=O), 153.5 (C-7a), 152.3(C-6), 151.6 (C-3), 143.8 (OㄷH=CH), 128.8 (C-7), 110.8 (C-3a), 110.5 (C-5), 106.7 (OCH=ㅡㅐ), 61.0 (OMe), 60.9 (OMe), 33.2 (Me); HR-ESIMS: $m / z$ 237.0759 [M+H] ${ }^{+}$calcd for $\mathrm{C}_{12} \mathrm{H}_{12} \mathrm{O}_{5}+\mathrm{H}^{+}(237.0757) .{ }^{15}$

Synthesis of furanochalcones 3a-v. Khellinone (80 mg, $0.338 \mathrm{mmol})$ was reacted with different aromatic aldehydes (1.2 equiv.) in the presence of catalytic amount of $1 \mathrm{M}$ of $\mathrm{KOH}$ in $50 \mathrm{ml}$ methanol at a temperature of $0{ }^{\circ} \mathrm{C}$ to $1{ }^{\circ} \mathrm{C}$ over a period of $12-14 \mathrm{~h}$. After completion of the reaction, methanol was evaporated over vacuo rotavapor and the obtained residue was partitioned in between dichloromethane and water. The dichloromethane layer was washed with brine solution and was dried over anhydrous 
sodium sulphate. The solvent was evaporated over vacuo rotavapor and the obtained crude product was purified using silica gel column chromatography (5-25\%) to get furanochalcones 3a-v in 40-85\% yield. (1-(6-Hydroxy-4,7-dimethoxy-benzofuran-5-yl)-3-phenyl-propenone) (3a): yellow orange solid; HPLC: $\mathrm{t}_{\mathrm{R}}=4.7 \mathrm{~min}$ (99\% purity); yield: 41\%; m.p. 123-126 ${ }^{\circ} \mathrm{C}$; IR $\left(\mathrm{CHCl}_{3}\right): v_{\max } 3860,3791,3697$, 3436, 3060, 2930, 2850, 1630, 1606, 1559, 1494, 1446, $1360 \mathrm{~cm}^{-1} ;{ }^{1} \mathrm{H}$ NMR (400 MHz, $\left.\mathrm{CDCl}_{3}\right): \delta$ (ppm) 7.88 (d, J = 2.2 Hz, 2H, CH), 7.64-7.67 (m, 2H), 7.53 (d, J = 2.2 Hz, 1H, OC프=CH), 7.42-7.45 (m, 3H), 6.89 (d, J = 4.0 Hz, 1H, OCH=C $\underline{H}), 4.09$ (s, 3H, OMe), 4.05 (s, 3H, OMe); ${ }^{13} \mathrm{C}$ NMR (100

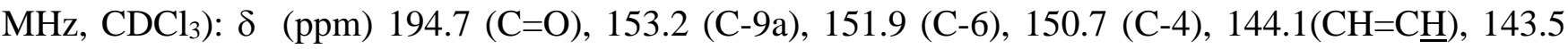
$(\mathrm{OC} \underline{\mathrm{H}}=\mathrm{CH}), 135.1,130.4,129.6,129.0,128.5,127.0,112.8,111.9,105.2(\mathrm{OCH}=\underline{\mathrm{CH}}), 62.0,61.0$; HRESIMS: m/z $325.1078[\mathrm{M}+\mathrm{H}]^{+}$calcd for $\mathrm{C}_{19} \mathrm{H}_{16} \mathrm{O}_{5}+\mathrm{H}^{+}(325.1075) .{ }^{16}$

3-(4-Bromo-phenyl)-1-(6-hydroxy-4,7-dimethoxy-benzofuran-5-yl)-popenone) (3b): $\quad$ white solid; HPLC: $t_{\mathrm{R}}=4.5 \min \left(100 \%\right.$ purity); yield: 52\%; m.p. $135-137{ }^{\circ} \mathrm{C}$; IR $\left(\mathrm{CHCl}_{3}\right): v_{\max } \quad 3400, \quad 2919$, 2850, 1682, 1613, 1544, 1414, 1389, 1435, 1349, 1279, 112, $\left.1012 \mathrm{~cm}^{-1} ;{ }^{1} \mathrm{H} \mathrm{NMR} 400 \mathrm{MHz}, \mathrm{CDCl}_{3}\right): \delta$ (ppm) $7.82(\mathrm{~d}, J=12.0 \mathrm{~Hz}, 2 \mathrm{H}, \mathrm{CH}), 7.58(\mathrm{~d}, J=8.0 \mathrm{~Hz}, 1 \mathrm{H}, \mathrm{CH}), 7.53(\mathrm{~d}, J=4.0 \mathrm{~Hz}, 1 \mathrm{H}, \mathrm{OC} \underline{\mathrm{H}}=\mathrm{CH})$, 7.40 (d, $J=12.0 \mathrm{~Hz}, 2 \mathrm{H}, \mathrm{CH}), 6.89$ (d, $J=4.0 \mathrm{~Hz}, 1 \mathrm{H}, \mathrm{OCH}=\mathrm{C} \underline{\mathrm{H}}), 4.09$ (s, 3H, OMe), 4.04 (s, 3H, OMe); ${ }^{13} \mathrm{C}$ NMR (100 MHz, $\left.\mathrm{CDCl}_{3}\right): \delta$ (ppm) 194.4 (C=O), 153.1 (C-6), 152.1 (C-9a), 150.7 (C-4), $144.3(\mathrm{OC} H=\mathrm{CH}), 141.4,137.3,133.1,130.9,130.5,127.2,123.1,112.7,111.7,106.2(\mathrm{OCH}=\underline{\mathrm{C}} \mathrm{H})$, 62.0, 61.0; HR-ESIMS m/z $403.0175[\mathrm{M}+\mathrm{H}]^{+}$calcd for $\mathrm{C}_{19} \mathrm{H}_{15} \mathrm{BrO}_{5}+\mathrm{H}^{+}$(403.0175).

\section{3-(4-Chlorophenyl)-1-(6-hydroxy-4,7-dimethoxybenzofuran-5-yl)prop-2-en-1-one $\quad$ (3c): orange} crystals; HPLC: $t_{\mathrm{R}}=49.6 \min \left(90 \%\right.$ purity); yield: 48\%; m.p. $152-154{ }^{\circ} \mathrm{C}$; IR $\left(\mathrm{CHCl}_{3}\right): v_{\max } 3448,3053$, 2928, 2868, 2304, 1730, 1656, 1619, 1585, 1462, 1386, 1327, 1313, 12679, 1262, 1210, 1149, $1039 \mathrm{~cm}^{-}$ 1; ${ }^{1} \mathrm{H}$ NMR (400 MHz, $\left.\mathrm{CDCl}_{3}\right): \delta(\mathrm{ppm}) 7.98(\mathrm{~d}, J=4.0 \mathrm{~Hz}, 2 \mathrm{H}, \mathrm{CH}), 7.52(\mathrm{~d}, J=4.0 \mathrm{~Hz}, 1 \mathrm{H}$, OCH$=C H), 7.40$ (d, $J=8.0 \mathrm{~Hz}, 2 \mathrm{H}, \mathrm{CH}), 7.19(\mathrm{~m}, 2 \mathrm{H}, \mathrm{CH}), 6.88(\mathrm{~d}, J=4.0 \mathrm{~Hz}, 1 \mathrm{H}, \mathrm{OCH}=\mathrm{C} \underline{\mathrm{H}}), 4.09$ (s, 3H, OMe), 4.05 (s, 3H, OMe); $\left.{ }^{13} \mathrm{C} \mathrm{NMR} \mathrm{(100} \mathrm{MHz,} \mathrm{CDCl}\right): \delta$ (ppm) 194.5 (C=O), 153.2 (C-6), 152.0 (C-9a), 150.7 (C-4), 144.2 (OㄷH=CH), 141.9, 136.3, 133.6, 129.6, 129.3, 127.5, 127.4 112.7, 
111.8, 106.2 $(\mathrm{OCH}=\underline{\mathrm{CH}}), 62.0,61.0$; HR-ESIMS: $\mathrm{m} / \mathrm{z} 359.0677[\mathrm{M}+\mathrm{H}]^{+}$calcd for $\mathrm{C}_{19} \mathrm{H}_{15} \mathrm{ClO}_{5}+\mathrm{H}^{+}$ $(359.0608){ }^{26}$

$$
\text { 3-(3-Bromo-4-fluorophenyl)-1-(6-hydroxy-4,7-dimethoxybenzofuran-5-yl)prop-2-en-1-one) }
$$

white solid; HPLC: $t_{\mathrm{R}}=5.0$ min (97\% purity); yield: $44 \%$; m.p.186-188 ${ }^{\circ} \mathrm{C}$; IR $\left(\mathrm{CHCl}_{3}\right): v_{\max } 3400$, 2921, 2850, 1630, 1557, 1494, 1463, 1442, 1417, 1382, 1359, 1332, 1299, 1269, 1151, $1091 \mathrm{~cm}^{-1} ;{ }^{1} \mathrm{H}$ NMR (400 MHz, $\left.\mathrm{CDCl}_{3}\right): \delta(\mathrm{ppm}) 7.85(\mathrm{~m}, 1 \mathrm{H}, \mathrm{CH}), 7.76(\mathrm{~d}, J=8.0 \mathrm{~Hz}, 2 \mathrm{H}, \mathrm{CH}), 7.54(\mathrm{t}, \quad J=4.0$ Hz, 2H, CH), 7.12 (t, $J=12.0 \mathrm{~Hz}, 1 \mathrm{H}, \mathrm{CH}), 6.89$ (d, $J=2.2 \mathrm{~Hz}, 1 \mathrm{H}, \mathrm{OCH}=\mathrm{CH}), 4.09$ (s, 3H, OMe), 4.05 (s, 3H, OMe); ${ }^{13} \mathrm{C}$ NMR (100 MHz, $\left.\mathrm{CDCl}_{3}\right): \delta(\mathrm{ppm}) 194.5(\mathrm{C}=\mathrm{O}), 165.3\left(\mathrm{~d},{ }^{1} J_{C F}=251 \mathrm{~Hz}\right)$, 162.8, 153.1, 151.9, 150.6, $144.2(\mathrm{O} \underline{\mathrm{CH}}=\mathrm{CH}), 142.2,131.4,130.4\left(\mathrm{~d},{ }^{3} J_{C F}=8.0 \mathrm{~Hz}\right), 130.3,129.6$, 126.7, $116.2\left({ }^{2} J_{C F}=22.0 \mathrm{~Hz}\right), 112.8,111.9,106.1(\mathrm{OCH}=\underline{\mathrm{CH}}), 62.0,61.0 ;$ HR-ESIMS: $\mathrm{m} / \mathrm{z} 423.0060$ $[\mathrm{M}+\mathrm{H}]^{+}$calcd for $\mathrm{C}_{19} \mathrm{H}_{14} \mathrm{BrFO}_{5}+\mathrm{H}^{+}$(423.0060).

3-(4-Fluro-phenyl)-1-(6-hydroxy-4,7-dimethoxy-benzofuran-5-yl)-propenone (3e): cream colored oil; HPLC: $t_{\mathrm{R}}=48.4$ min (98\% purity); yield: 40\%; m.p.129-130 ${ }^{\circ} \mathrm{C}$; IR $\left(\mathrm{CHCl}_{3}\right): v_{\max } 3400,2922,2851$, 1628, 1601, 1556, 1544, 1510, 1461, 1443, 1413, 1360, 1297, 1299, 1266, 1151, $1091 \mathrm{~cm}^{-1}$; ${ }^{1} \mathrm{H}$ NMR (400 MHz, $\left.\mathrm{CDCl}_{3}\right): \delta(\mathrm{ppm}) 7.82(\mathrm{~s}, 2 \mathrm{H}, \mathrm{CH}), 7.64(\mathrm{dd}, J=8.0,5.5 \mathrm{~Hz}, 2 \mathrm{H}, \mathrm{CH}), 7.53(\mathrm{~d}, J=2.2 \mathrm{~Hz}$, 1H, OCH=CH), 7.12 (t, $J=8.6 \mathrm{~Hz}, 2 \mathrm{H}, \mathrm{CH}), 6.88(\mathrm{~d}, J=2.3 \mathrm{~Hz}, 1 \mathrm{H}, \mathrm{OCH}=\mathrm{C} \underline{\mathrm{H}}), 4.09$ (s, 3H, OMe), 4.05 (s, 3H, OMe); ${ }^{13} \mathrm{C}$ NMR (100 MHz, $\left.\mathrm{CDCl}_{3}\right): \delta(\mathrm{ppm}) 194.5(\mathrm{C}=\mathrm{O}), 165.0\left(\mathrm{~d},{ }^{1} J_{\mathrm{CF}}=251.2 \mathrm{~Hz}\right)$, 163.0, 153.1, 151.9, 150.7, 144.2 (OㅡH=CH), 142.2, 131.4, $130.4\left(\mathrm{~d},{ }^{3} J_{\mathrm{CF}}=8.75 \mathrm{~Hz}\right), 126.7\left(\mathrm{~d},{ }^{4} J_{\mathrm{CF}}=\right.$ $3.0 \mathrm{~Hz}), 116.2\left(\mathrm{~d},{ }^{2} J_{\mathrm{CF}}=22.5 \mathrm{~Hz}\right), 112.8,111.8,106.2(\mathrm{OCH}=\underline{\mathrm{CH}}), 62.0,61.0 ; \mathrm{HR}-\mathrm{ESIMS}: \mathrm{m} / \mathrm{z}$ $365.0838[\mathrm{M}+\mathrm{Na}]^{+}$calcd for $\mathrm{C}_{19} \mathrm{H}_{15} \mathrm{FNaO}_{5}(365.0801) .{ }^{27}$

3-(2,4-Difluro-phenyl)-1-(6-hydroxy-4,7-dimethoxy-benzofuran-5-yl)-propenone) (3f): white solid; HPLC: $t_{\mathrm{R}}=42.4$ min (98\% purity); yield: 40\%; m.p. 154-156 $\mathrm{C}$; IR $\left(\mathrm{CHCl}_{3}\right): v_{\max } 3400,2919,2850$, 1633, 1618, 1588, 1562, 1542, 1464, 1438, 1412, 1377, 1357, 1286, 1211, 1153, $1119 \mathrm{~cm}^{-1} ;{ }^{1} \mathrm{H}$ NMR (400 MHz, $\left.\mathrm{CDCl}_{3}\right): \delta(\mathrm{ppm}) 7.83(\mathrm{~d}, J=16.0 \mathrm{~Hz}, 1 \mathrm{H}, \mathrm{CH}), 7.72(\mathrm{~d}, J=12.0 \mathrm{~Hz}, 1 \mathrm{H}, \mathrm{CH}), 7.54(\mathrm{~d}, J=$ $2.2 \mathrm{~Hz}, 1 \mathrm{H}, \mathrm{OC} \underline{\mathrm{H}}=\mathrm{CH}), 7.14(\mathrm{~d}, J=4.0 \mathrm{~Hz}, 2 \mathrm{H}, \mathrm{CH}), 6.89(\mathrm{~d}, J=4.0 \mathrm{~Hz}, 1 \mathrm{H}, \mathrm{CH}), 6.86(\mathrm{~d}, J=4.0 \mathrm{~Hz}$, 1H, OCH=CH), 4.09 (s, 3H, OMe), 4.07 (s, 3H, OMe); ${ }^{13} \mathrm{C}$ NMR (100 MHz, CDCl $): \delta(p p m) 194.2$ 
$(\mathrm{C}=\mathrm{O}), 164.8,162.0,153.2,152.2,150.8,144.3(\mathrm{O} \underline{\mathrm{CH}}=\mathrm{CH}), 140.2,138.5,129.5,112.5,111.6,111.0$, 110.9, 110.7, 105.4, $105.2(\mathrm{OCH}=\underline{\mathrm{CH}}), 61.9,61.0 ;$ HR-ESIMS: $\mathrm{m} / \mathrm{z} 361.0912[\mathrm{M}+\mathrm{H}]^{+}$calcd for $\mathrm{C}_{19} \mathrm{H}_{14} \mathrm{~F}_{2}, \mathrm{O}_{5}+\mathrm{H}^{+}(361.0882)$.

1-(6-Hydroxy-4,7-dimethoxybenzofuran-5-yl)-3-(pyridin-3-yl)prop-2-en-1-one (3g): yellow orange crystals; HPLC: $t_{\mathrm{R}}=49.6$ min (95\%); yield: 85\%; m.p. 112-114 ${ }^{\circ} \mathrm{C}$; IR $\left(\mathrm{CHCl}_{3}\right): 3400,2919,2850$, 1633, 1618, 1588, 1562, 1542, 1464, 1438, 1412, 1377, 1357, 1286, 1211, 1153, $1119 \mathrm{~cm}^{-1} ;{ }^{1} \mathrm{H}$ NMR (400 MHz, $\left.\mathrm{CDCl}_{3}\right): \delta(\mathrm{ppm}) 8.88$ (s, 1H, CH ), 8.63 (d, $\left.J=4.0 \mathrm{~Hz}, 1 \mathrm{H}, \mathrm{CH}\right), 7.94$ (m, 2H, CH), 7.81 (d, $J=16.0 \mathrm{~Hz}, 1 \mathrm{H}, \mathrm{CH}$ ), 7.54 (d, $J=2.3 \mathrm{~Hz}, 1 \mathrm{H}, \mathrm{OC} \underline{\mathrm{H}}=\mathrm{CH}$ ), 7.37 (dd, $J=8.0,4.9 \mathrm{~Hz}, 1 \mathrm{H}, \mathrm{CH}), 6.89$ (d, $J=2.3 \mathrm{~Hz}, 1 \mathrm{H}, \mathrm{OCH}=\mathrm{CH}$ ), 4.09 (s, 3H, OMe), 4.06 (s, 3H, OMe); ${ }^{13} \mathrm{C}$ NMR $\left(100 \mathrm{MHz}, \mathrm{CDCl}_{3}\right): \delta$ (ppm) 194.2 (C=O), 153.2, 152.2, 150.9, 149.8, 144.3, 139.3, 134.8, 131.0, 128.9, 123.8, 112.5, 112.5, 111.6, 111.5, 106.3, 61.8, 61.1; HR-ESIMS: $\mathrm{m} / \mathrm{z} 326.1033[\mathrm{M}+\mathrm{H}]^{+}$calcd for $\mathrm{C}_{18} \mathrm{H}_{15} \mathrm{NO}_{5}+\mathrm{H}^{+}$ (326.1023).

1-(6-Hydroxy-4,7-dimethoxybenzofuran-5-yl)-3-(4-methoxyphenyl)prop-2-en-1-one (3h): yellow crystals; HPLC: $t_{\mathrm{R}}=5.3 \mathrm{~min}\left(100 \%\right.$ purity); yield: 52\%; m.p. $148-149^{\circ} \mathrm{C}$ IR $\left(\mathrm{CHCl}_{3}\right): v_{\max } 3435,2923$, 2851, 1630, 1606, 1564, 1543, 1456, 1438, 1422, 1404, 1358, 1306, 1293, 1250, 1153, $1119 \mathrm{~cm}^{-1} ;{ }^{1} \mathrm{H}$ NMR (400 MHz, CDCl $): \delta(\mathrm{ppm}) 7.84(\mathrm{~d}, J=16.0 \mathrm{~Hz}, 2 \mathrm{H}, \mathrm{CH}), 7.62(\mathrm{~d}, J=4.0 \mathrm{~Hz}, 2 \mathrm{H}, \mathrm{CH}), 7.53$ (d, $J=2.3 \mathrm{~Hz}, 1 \mathrm{H}, \mathrm{OC} \underline{\mathrm{H}}=\mathrm{CH}), 7.30$ (d, $J=12.0 \mathrm{~Hz}, 1 \mathrm{H}, \mathrm{CH}), 6.95$ (d, $J=4.0 \mathrm{~Hz}, 2 \mathrm{H}, \mathrm{CH}), 6.89$ (d, $J=$ $12.0 \mathrm{~Hz}, 1 \mathrm{H}, \mathrm{CH}$ ), 6.88 (d, $J=4.0 \mathrm{~Hz}, 1 \mathrm{H}, \mathrm{OCH}=\mathrm{CH}$ ), 4.09 (s, 3H, OMe), 4.04 (s, 3H, OMe), 3.87 (s, 3H, OMe); ${ }^{13} \mathrm{C}$ NMR (100 MHz, $\left.\mathrm{CDCl}_{3}\right): \delta$ (ppm) 194.6 (C=O), 161.7, 153.2, 151.7, 150.6, 144.1 (OCLH=CH), 143.8, 130.3, 127.8, 124.5, 114.5, 113.4, 112.9, 112.0, $105.1(\mathrm{OCH}=\underline{\mathrm{CH}}), 62.0,61.0,55.4$; HR-ESIMS: $m / z 355.1171[\mathrm{M}+\mathrm{H}]^{+}$calcd for $\mathrm{C}_{20} \mathrm{H}_{18} \mathrm{O}_{6}+\mathrm{H}^{+}$(354.1103). ${ }^{27}$

3-(2,6-Dichlorophenyl)-1-(6-hydroxy-4,7-dimethoxybenzofuran-5-yl)prop-2-en-1-one (3i): orange yellow crystals; HPLC: $t_{\mathrm{R}}=5.3 \mathrm{~min}\left(100 \%\right.$ purity); yield: $57 \%$; m.p. $298-299{ }^{\circ} \mathrm{C} ; \mathrm{IR}\left(\mathrm{CHCl}_{3}\right): v_{\max }$ 3399, 3161, 3090, 2951, 2921, 2851, 1640, 1613, 1577, 1472, 1441, 1427, 1378, 1357, 1328, 1301, 1275, 1242, 1213, 1185, $1145 \mathrm{~cm}^{-1}$; ${ }^{1} \mathrm{H}$ NMR (400 MHz, $\left.\mathrm{CDCl}_{3}\right): \delta(\mathrm{ppm}) 7.98$ (d, $J=8.0 \mathrm{~Hz}, 2 \mathrm{H}$, CH), 7.52 (d, $J=2.3 \mathrm{~Hz}, 1 \mathrm{H}, \mathrm{OC} \underline{\mathrm{H}}=\mathrm{CH}), 7.40(\mathrm{~d}, J=8.0 \mathrm{~Hz}, 2 \mathrm{H}, \mathrm{CH}), 7.23$ (d, $J=8.0 \mathrm{~Hz}, 1 \mathrm{H}, \mathrm{CH})$, 
$6.88(\mathrm{~d}, J=4.0 \mathrm{~Hz}, 1 \mathrm{H}, \mathrm{OCH}=\mathrm{C} \underline{\mathrm{H}}), 4.09$ (s, 3H, OMe), 4.07 (s, 3H, OMe); ${ }^{13} \mathrm{C} \mathrm{NMR}(100 \mathrm{MHz}$, $\left.\mathrm{CDCl}_{3}\right): \delta(\mathrm{ppm}) 194.4(\mathrm{C}=\mathrm{O}), 153.4,152.2,151.1,143.9(\mathrm{OC} H=\mathrm{CH}), 141.7,136.3,135.3,134.7$, 132.6, 129.9, 129.3, 128.8, 112.3, 111.5, 105.3 (OCH=ㅡㅐ), 61.9 (OMe), 61.1 (OMe); HR-ESIMS: m/z 393.0287 $[\mathrm{M}+\mathrm{H}]^{+}$calcd for $\mathrm{C}_{19} \mathrm{H}_{14} \mathrm{Cl}_{2}, \mathrm{O}_{5}+\mathrm{H}^{+}(393.0291)$.

\section{3-(5-Bromo-2-ethoxyphenyl)-1-(6-hydroxy-4,7-dimethoxybenzofuran-5-yl)prop-2-en-1-one}

(3j):

orange powder; HPLC: $t_{\mathrm{R}}=5.1 \mathrm{~min}$ (100\% purity); yield: 55\%; m.p. $260-261{ }^{\circ} \mathrm{C}$; IR $\left(\mathrm{CHCl}_{3}\right): v_{\max }$ 3399, 3161, 3090, 2951, 2921, 2851, 1640, 1613, 1577, 1472, 1441, 1427, 1378, 1357, $1328 \mathrm{~cm}^{-1} ;{ }^{1} \mathrm{H}$ NMR (400 MHz, CDCl $): \delta(\mathrm{ppm}) 8.12$ (d, $J=16.0 \mathrm{~Hz}, 1 \mathrm{H}, \mathrm{CH}), 7.90$ (d, $J=16.0 \mathrm{~Hz}, 1 \mathrm{H}, \mathrm{CH}), 7.73$ (d, $J=4.0 \mathrm{~Hz}, 1 \mathrm{H}, \mathrm{CH}), 7.53$ (d, $J=2.2 \mathrm{~Hz}, 1 \mathrm{H}, \mathrm{OC} \underline{\mathrm{H}}=\mathrm{CH}), 7.43$ (d, $J=4.0 \mathrm{~Hz}, 1 \mathrm{H}, \mathrm{CH}), 6.88$ (d, $J=$ $2.2 \mathrm{~Hz}, 1 \mathrm{H}, \mathrm{OCH}=\mathrm{C} \underline{\mathrm{H}}), 6.82$ (d, $J=8.0 \mathrm{~Hz}, 1 \mathrm{H}, \mathrm{CH}), 4.13$ (m, 2H, CH ), 4.09 (s, 3H, OMe), 4.04 (s, 3H, OMe), 1.50 (t, $J=7.0 \mathrm{~Hz}, 3 \mathrm{H}, \mathrm{Me}) ;{ }^{13} \mathrm{C} \mathrm{NMR}\left(100 \mathrm{MHz}, \mathrm{CDCl}_{3}\right): \delta(\mathrm{ppm}) 194.7(\mathrm{C}=\mathrm{O}), 157.1$, 157.0, 153.2, 151.9, 150.8, $144.2(\mathrm{O} \underline{\mathrm{CH}}=\mathrm{CH}), 137.2,134.0,130.9,128.1,126.1,113.9,112.8,112.8$, 111.9, $106.2(\mathrm{OCH}=\underline{\mathrm{C}} \mathrm{H}), 64.4,62.0,61.0,14.7$; HR-ESIMS: $\mathrm{m} / \mathrm{z} 447.0433[\mathrm{M}+\mathrm{H}]^{+}$calcd for $\mathrm{C}_{21} \mathrm{H}_{19} \mathrm{BrO}_{6}+\mathrm{H}^{+}$(447.0437).

3-(2-Chlorophenyl)-1-(6-hydroxy-4,7-dimethoxybenzofuran-5-yl)prop-2-en-1-one $\quad$ (3k): $\quad$ yellow powder; HPLC: $t_{\mathrm{R}}=5.1$ min (100\% purity); yield: 55\%; m.p. 150-151 ${ }^{\circ} \mathrm{C}$; IR $\left(\mathrm{CHCl}_{3}\right): v_{\max } 3435,2922$, 2851, 2650, 2342, 1693, 1628, 1591, 1571, 1469, 1439, 1408, 1363, $1316 \mathrm{~cm}^{-1} ;{ }^{1} \mathrm{H}$ NMR (400 MHz, $\left.\mathrm{CDCl}_{3}\right): \delta(\mathrm{ppm}) 8.23(\mathrm{~d}, J=16.0 \mathrm{~Hz}, 1 \mathrm{H}, \mathrm{CH}), 7.86(\mathrm{~d}, J=8.0 \mathrm{~Hz}, 1 \mathrm{H}, \mathrm{CH}), 7.74(\mathrm{~d}, J=2.2 \mathrm{~Hz}, 1 \mathrm{H}$, $\mathrm{CH}), 7.53$ (d, $J=2.3 \mathrm{~Hz}, 1 \mathrm{H}, \mathrm{OC} \underline{\mathrm{H}}=\mathrm{CH}), 7.33$ (dd, $J=2.3,4.0 \mathrm{~Hz}, 2 \mathrm{H}, \mathrm{CH}), 6.88(\mathrm{~d}, J=2.3 \mathrm{~Hz}, 1 \mathrm{H}$, $\mathrm{OCH}=\mathrm{C} \underline{\mathrm{H}}$ ), 4.09 (s, 3H, OMe), 4.05 (s, 3H, OMe); ${ }^{13} \mathrm{C}$ NMR (100 MHz, $\left.\mathrm{CDCl}_{3}\right): \delta$ (ppm) $194.4(\mathrm{C}=\mathrm{O})$, 153.1, 152.1, 150.7, 144.3 (OCLH=CH), 141.1, 137.3, 133.1, 130.9, 130.5, 129.5, 128.3, 127.2, 123.1, 112.7, 111.7, 105.2, 62.0, 61.0; HR-ESIMS: $\mathrm{m} / \mathrm{z} 359.0680[\mathrm{M}+\mathrm{H}]^{+}$calcd for $\mathrm{C}_{19} \mathrm{H}_{15} \mathrm{ClO}_{5}+\mathrm{H}^{+}$ $(359.0680){ }^{26}$

3-(4,7-dimethoxy-6-hydroxybenzofuran-5-yl)-1-(2-3-dimethoxyphenyl)-3-oxopropene (3l): HPLC: $t_{\mathrm{R}}$ $=4.7$ min (100\% purity); yield: 57\%; m.p. 260-262 ${ }^{\circ} \mathrm{C}$; IR $\left(\mathrm{CHCl}_{3}\right): v_{\max } 3400,2923,2851,1627,1561$, 1511, 1463, 1439, 1383, 1360, 1301, 1264, 1064, $1022 \mathrm{~cm}^{-1} ;{ }^{1} \mathrm{H}$ NMR (400 MHz, CDCl $): \delta(p p m) 7.83$ 
(d, $J=16.0 \mathrm{~Hz}, 2 \mathrm{H}, \mathrm{CH}), 7.53$ (d, $J=4.0 \mathrm{~Hz}, 1 \mathrm{H}, \mathrm{OC} \underline{\mathrm{H}}=\mathrm{CH}), 7.25$ (d, $J=2.2 \mathrm{~Hz}, 2 \mathrm{H}, \mathrm{CH}), 7.16(\mathrm{~d}, J=$ $4.0 \mathrm{~Hz}, 1 \mathrm{H}, \mathrm{CH}), 6.91$ (d, $J=12.0 \mathrm{~Hz}, 1 \mathrm{H}, \mathrm{CH}), 6.87$ (d, $J=4.0 \mathrm{~Hz}, 1 \mathrm{H}, \mathrm{OCH}=\mathrm{CH}), 4.09$ (s, 3H, OMe), 4.03 (s, 3H, OMe), 3.95 (s, 3H, OMe), 3.93 (s, 3H, OMe); $\left.{ }^{13} \mathrm{C} \mathrm{NMR} \mathrm{(100} \mathrm{MHz,} \mathrm{CDCl}\right): \delta(\mathrm{ppm})$ $195.6(\mathrm{C}=\mathrm{O}), 163.8,161.1,154.0,152.2,151.3,144.7,140.3,131.4,130.2,125.3,125.2,118.0,113.6$, 112.9, 106.2, 105.8, 62.7, 61.7, 56.2, 56.2; HR-ESIMS: $\mathrm{m} / \mathrm{z} \quad 385.1278[\mathrm{M}+\mathrm{H}]^{+}$calcd for $\mathrm{C}_{21} \mathrm{H}_{20} \mathrm{O}_{7}+$ $\mathrm{H}^{+}(385.1281)$.

3-(4,7-Dimethoxy-6-hydroxybenzofuran-5-yl)-1-(2-4-dimethoxyphenyl)-3-oxopropene (3m): colorless oil; HPLC: $t_{\mathrm{R}}=4.7 \mathrm{~min}\left(100 \%\right.$ purity); yield: 55\%; m.p. $264-266{ }^{\circ} \mathrm{C}$; IR $\left(\mathrm{CHCl}_{3}\right): v_{\max } 3400,2923$, 2851, 1627, 1561, 1511, 1463, 1439, 1383, 1360, 1301, 1264, 1064, $1022 \mathrm{~cm}^{-1}$; ${ }^{1} \mathrm{H}$ NMR (400 MHz, $\left.\mathrm{CDCl}_{3}\right): \delta(\mathrm{ppm}) 8.21(\mathrm{~d}, J=16.0 \mathrm{~Hz}, 1 \mathrm{H}, \mathrm{CH}), 7.93(\mathrm{~d}, J=16.0 \mathrm{~Hz}, 1 \mathrm{H}, \mathrm{CH}), 7.61(\mathrm{~d}, J=8.0 \mathrm{~Hz}, 1 \mathrm{H}$, $\mathrm{CH}), 7.51$ (d, $J=2.2 \mathrm{~Hz}, 1 \mathrm{H}, \mathrm{OC} \underline{\mathrm{H}}=\mathrm{CH}), 6.87$ (s, 1H, CH), 6.53 (d, $J=4.0 \mathrm{~Hz}, 1 \mathrm{H}, \mathrm{OCH}=\mathrm{C} \underline{\mathrm{H}}), 6.48$ (s, 1H, CH), 4.09 (s, 3H, OMe), 3.91 (s, 3H, OMe), 3.87 (s, 3H, OMe); ${ }^{13} \mathrm{C}$ NMR (100 MHz, $\left.\mathrm{CDCl}_{3}\right): \delta$ (ppm) 194.9 (C=O), 163.2, 160.5, 153.3, 151.5, 150.6, 144.0, 139.6, 130.7, 129.4, 124.6, 117.3, 112.9, 112.2, 105.5, 105.2, 98.4, 62.0, 61.0, 55.5, 55.5; HR-ESIMS: $m / z$ 385.1276 $[\mathrm{M}+\mathrm{H}]^{+}$calcd for $\mathrm{C}_{21} \mathrm{H}_{20} \mathrm{O}_{7}+$ $\mathrm{H}^{+}(385.1281){ }^{26}$

1-(6-Hydroxy-4,7-dimethoxybenzofuran-5-yl)-3-(perfluorophenyl)prop-2-en-1-one (3n): white solid; HPLC: $t_{\mathrm{R}}=5.0 \mathrm{~min}\left(92 \%\right.$ purity); yield: 50\%; m.p. $112-114{ }^{\circ} \mathrm{C} ; \mathrm{IR}\left(\mathrm{CHCl}_{3}\right): v_{\max } 3400,2924,2853$, 1726, 1656, 1500, 1462, 1385, 1280, 1209, 1151, 1053, $1021 \mathrm{~cm}^{-1} ;{ }^{1} \mathrm{H}$ NMR (400 MHz, $\left.\mathrm{CDCl}_{3}\right): \delta$ (ppm) 8.12 (d, $J=16.0 \mathrm{~Hz}, 1 \mathrm{H}, \mathrm{CH}), 7.81$ (d, $J=16.0 \mathrm{~Hz}, 1 \mathrm{H}, \mathrm{CH}), 7.53$ (d, $J=4.0 \mathrm{~Hz}, 1 \mathrm{H}$, $\mathrm{OC} \underline{\mathrm{H}}=\mathrm{CH}), 6.91$ (d, $J=4.0 \mathrm{~Hz}, 1 \mathrm{H}, \mathrm{OCH}=\mathrm{C} \underline{\mathrm{H}}), 4.17$ (s, 3H, OMe), 4.11 (s, 3H, OMe); ${ }^{13} \mathrm{C}$ NMR (100 $\left.\mathrm{MHz}, \mathrm{CDCl}_{3}\right): \delta(\mathrm{ppm}) 194.3(\mathrm{C}=\mathrm{O}), 153.5,152.2,151.0,144.9,144.1(\mathrm{OC} H=\mathrm{CH}), 133.0,132.9,129.3$, 127.2, 112.1, 111.3, 108.6, $105.1(\mathrm{OCH}=\underline{\mathrm{CH}}), 61.3,61.0$; HR-ESIMS: $m / \mathrm{z} 415.0603[\mathrm{M}+\mathrm{H}]^{+}$calcd for $\mathrm{C}_{19} \mathrm{H}_{11} \mathrm{~F}_{5} \mathrm{O}_{5}+\mathrm{H}^{+}$(415.0599).

$$
\text { 1-(6-Hydroxy-4,7-dimethoxybenzofuran-5-yl)-3-(2,4,5-trimethoxyphenyl)prop-2-en-1-one }
$$

(3o): reddish orange crystals; HPLC: $t_{\mathrm{R}}=8.7 \mathrm{~min}$ (100\% purity); yield: 50\%; m.p. $131-133{ }^{\circ} \mathrm{C}$; IR $\left(\mathrm{CHCl}_{3}\right)$ : $v_{\max } 3434,2930,2867,1724,1656,1517,1463,1385,1263,1210,1159,1084,1024 \mathrm{~cm}^{-1} ;{ }^{1} \mathrm{H}$ NMR 
(400 MHz, $\left.\mathrm{CDCl}_{3}\right): \delta(\mathrm{ppm}) 8.29$ (d, $\left.J=16.0 \mathrm{~Hz}, 1 \mathrm{H}, \mathrm{CH}\right), 7.92$ (d, $\left.J=16.0 \mathrm{~Hz}, 1 \mathrm{H}, \mathrm{CH}\right), 7.59$ (s, $1 \mathrm{H}$, $\mathrm{OC} \underline{\mathrm{H}}=\mathrm{CH}), 7.22(\mathrm{~m}, 1 \mathrm{H}, \mathrm{CH}), 6.94(\mathrm{~s}, \mathrm{OCH}=\mathrm{C} \underline{\mathrm{H}}, 1 \mathrm{H}), 6.60(\mathrm{~s}, 1 \mathrm{H}, \mathrm{CH}), 4.16-3.97$ (m, 15H, OMe); ${ }^{13} \mathrm{C}$ NMR: (100 MHz, $\left.\mathrm{CDCl}_{3}\right): \delta$ (ppm) 194.7, 154.8, 153.2, 152.7, 151.5, 150.5, 144.0, 143.3, 139.3, 129.6, 124.4, 115.7, 113.0, 112.2, 111.2, 105.1, 96.7, 62.0 (OMe), 61.0 (OMe), 56.5 (OMe), 56.3 (OMe), 56.1 (OMe); HR-ESIMS: m/z $415.1390[\mathrm{M}+\mathrm{H}]^{+}$calcd for $\mathrm{C}_{22} \mathrm{H}_{21} \mathrm{O}_{8}+\mathrm{H}^{+}$(415.1390).

\section{3-(3-Bromo-4-methoxyphenyl)-1-(6-hydroxy-4,7-dimethoxybenzofuran-5-yl)prop-2-en-1-one}

(3p):

orange crystals; HPLC: $t_{\mathrm{R}}=5.4$ min (100\% purity); yield: 52\%; m.p. $261-263{ }^{\circ} \mathrm{C}$; IR $\left(\mathrm{CHCl}_{3}\right): v_{\max }$ 3454, 2927, 2866, 1730, 1654, 1590, 1464, 1386, 1365, 1326, 1312, 1279, 1102, 1084, $1048 \mathrm{~cm}^{-1} ;{ }^{1} \mathrm{H}$ NMR (400 MHz, $\left.\mathrm{CDCl}_{3}\right): \delta(\mathrm{ppm}) 8.11(\mathrm{~d}, J=16.0 \mathrm{~Hz}, 1 \mathrm{H}, \mathrm{CH}), 7.86(\mathrm{~d}, J=16.0 \mathrm{~Hz}, 1 \mathrm{H}, \mathrm{CH}), 7.73$ (s, 1H, CH), 7.52 (s, 1H, CH), 6.88 (s, 1H, CH), 6.84 (d, J = 8.0 Hz, 1H, CH), 4.09 (s, 3H, OMe), 4.06 (s, 3H, OMe), 3.91 (s, 3H, OMe); ${ }^{13} \mathrm{C}$ NMR (100 MHz, $\left.\mathrm{CDCl}_{3}\right): \delta$ (ppm) $194.8(\mathrm{C}=\mathrm{O}), 157.7,153.2$, 151.9, 150.7, 144.2, 137.1, 134.0, 134.0, 130.9, 128.3, 128.3, 126.1, 113.0, 112.7, 105.2, 62.0 (OMe), 61.0 (OMe), 55.8; HR-ESIMS: m/z 432.0300 [M+H] ${ }^{+}$calcd for $\mathrm{C}_{20} \mathrm{H}_{17} \mathrm{BrO}_{6}+\mathrm{H}^{+}$(432.0281).

3-(Benzo[d] [1,3] dioxol-5-yl)-1-(6-hydroxy-4,7-dimethoxybenzofuran-5-yl)prop-2-en-1-one

(3q): orange crystals; HPLC: $t_{\mathrm{R}}=5.6$ min (92\% purity); yield: 53\%; m.p. $248-251{ }^{\circ} \mathrm{C} ; \quad \mathrm{IR}\left(\mathrm{CHCl}_{3}\right): v_{\max }$ 3743, 3385, 3130, 2850, 1729, 1627, 1565, 1542, 1489, 1470, 1446, 1353, 1300, $125 \mathrm{~cm}^{-1}$; ${ }^{1} \mathrm{H}$ NMR $\left(400 \mathrm{MHz}, \mathrm{CDCl}_{3}\right): \delta(\mathrm{ppm}) 7.78(\mathrm{~d}, J=16.0 \mathrm{~Hz}, 2 \mathrm{H}, \mathrm{CH}), 7.53(\mathrm{~d}, J=4.0 \mathrm{~Hz}, 1 \mathrm{H}, \mathrm{OC} \underline{\mathrm{H}}=\mathrm{CH}), 7.15$ (m, 2H, CH), 6.88 (d, $J=2.2 \mathrm{~Hz}, 1 \mathrm{H}, \mathrm{OCH}=\mathrm{CH}), 6.87$ (d, $J=8.0 \mathrm{~Hz}, 1 \mathrm{H}, \mathrm{CH}), 6.04$ (s, 2H, CH 2$), 4.09$ (s, 3H, OMe), 4.04 (s, 3H, OMe); $\left.{ }^{13} \mathrm{C} \mathrm{NMR} \mathrm{(100} \mathrm{MHz,} \mathrm{CDCl}_{3}\right): \delta$ (ppm) 194.4 (C=O), 153.2, 151.7, 150.6, 149.9, 148.4, 144.1, 143.8, 143.6, 129.6, 125.4, 124.9, 112.8, 111.9, 108.7, 106.6, 105.2, 101.6, 62.0 (OMe), 61.0 (OMe); HR-ESIMS: m/z $369.0968[\mathrm{M}+\mathrm{H}]^{+}$calcd for $\mathrm{C}_{20} \mathrm{H}_{17} \mathrm{O}_{7}+\mathrm{H}^{+}(369.0954) .{ }^{26}$

1-(6-Hydroxy-4,7-dimethoxybenzofuran-5-yl)-3-(thiophen-3-yl)prop-2-en-1-one (3r): orange crystals; HPLC: $t_{\mathrm{R}}=3.9$ min (85\% purity); yield: 42\%; m.p. 139-141 ${ }^{\circ} \mathrm{C}$; IR $\left(\mathrm{CHCl}_{3}\right): v_{\max } 3584,3136,2922$, 2850, 1626, 1586, 1561, 1543, 1470, 1442, 1364, 1416, 1297, $1131 \mathrm{~cm}^{-1} ;{ }^{1} \mathrm{H}$ NMR (400 MHz, $\left.\mathrm{CDCl}_{3}\right)$ : $\delta(\mathrm{ppm}) 7.89(\mathrm{~d}, J=16.0 \mathrm{~Hz}, 1 \mathrm{H}, \mathrm{CH}), 7.73$ (d, $J=16.0 \mathrm{~Hz}, 1 \mathrm{H}, \mathrm{CH}), 7.62$ (s, CH, 1H), 7.52 (s, $1 \mathrm{H}$, CH), 7.40 (d, J = 8.0 Hz, 2H, CH), 6.87 (s, CH, 1H), 4.09 (s, 3H, OMe), 4.03 (s, 3H, OMe); ${ }^{13} \mathrm{C}$ NMR 
(100 MHz, $\left.\mathrm{CDCl}_{3}\right): \delta$ (ppm) 194.8 (C=O), 153.1, 151.8, 150.6, 144.1, 138.5, 137.2, 129.6, 129.1, 127.0, 126.6, 125.3, 112.8, 111.9, 105.1, 62.0 (OMe), 61.0 (OMe); HR-ESIMS: m/z 331.0619 [M+H] ${ }^{+}$calcd for $\mathrm{C}_{17} \mathrm{H}_{15} \mathrm{O}_{5} \mathrm{~S}+\mathrm{H}^{+}$(331.0634).

3-(2,4-Dichlorophenyl)-1-(6-hydroxy-4,7-dimethoxybenzofuran-5-yl)prop-2-en-1-on （3s): orange crystals; HPLC: $t_{\mathrm{R}}=5.0$ min (95\% purity); yield: 42\%; m.p. $148-149{ }^{\circ} \mathrm{C} ; \mathrm{IR}\left(\mathrm{CHCl}_{3}\right): v_{\max } 3399,3161$, 3090, 2951, 2921, 2851, 1640, 1613, 1577, 1472, 1441, 1427, 1378, 1357, 1328, 1301, 1275, 1242, 1213, 1185, $1145 \mathrm{~cm}^{-1} ;{ }^{1} \mathrm{H}$ NMR (400 MHz, $\left.\mathrm{CDCl}_{3}\right): \delta(\mathrm{ppm}) 8.14(\mathrm{~d}, J=16.0 \mathrm{~Hz}, 1 \mathrm{H}, \mathrm{CH}), 7.83(\mathrm{~d}, J$ $=16.0 \mathrm{~Hz}, 1 \mathrm{H}, \mathrm{CH}), 7.67(\mathrm{~d}, J=12.0 \mathrm{~Hz}, 1 \mathrm{H}, \mathrm{CH}), 7.53(\mathrm{~d}, J=4.0 \mathrm{~Hz}, 1 \mathrm{H}, \mathrm{OC} \underline{\mathrm{H}}=\mathrm{CH}), 7.48(\mathrm{~d}, J=3.0$ Hz, 1H, CH), 7.31 (m, 1H, CH), 6.88 (d, $J=2.2$ Hz, 1H, OCH=C파), 4.09 (s, 3H, OMe), 4.03 (s, 3H, OMe); ${ }^{13} \mathrm{C}$ NMR (100 MHz, $\left.\mathrm{CDCl}_{3}\right): \delta(\mathrm{ppm}) 194.2$ (C=O), 153.2, 152.1, 150.7, 144.3, 137.5, 136.3, 136.1, 132.0, 130.1, 129.8, 129.5, 128.4,127.6, 112.6, 111.7, 105.2, 61.9, 61.0; HR-ESIMS: m/z 393.0275 $[\mathrm{M}+\mathrm{H}]^{+}$calcd for $\mathrm{C}_{19} \mathrm{H}_{14} \mathrm{Cl}_{2} \mathrm{O}_{5}+\mathrm{H}^{+}$(393.0291).

1-(6-Hydroxy-4,7-dimethoxybenzofuran-5-yl)-3-(naphthalen-2-yl)prop-2-en-1-one （3t): orange crystals; HPLC: $t_{\mathrm{R}}=7.1$ min (99\% purity); yield: 42\%; m.p. 158-159 ${ }^{\circ} \mathrm{C}$; IR $\left(\mathrm{CHCl}_{3}\right): v_{\max } 3584,3136$, 2922, 2850, 1626, 1586, 1561, 1543, 1470, 1442, 1379, 1297, $1149 \mathrm{~cm}^{-1} ;{ }^{1} \mathrm{H}$ NMR (400 MHz, $\left.\mathrm{CDCl}_{3}\right)$ : $\delta(\mathrm{ppm}) 8.03(\mathrm{~m}, 3 \mathrm{H}, \mathrm{CH}), 7.86(\mathrm{~m}, 4 \mathrm{H}, \mathrm{CH}), 7.54$ (d, $J=2.2 \mathrm{~Hz}, 1 \mathrm{H}, \mathrm{OC} \underline{H}=\mathrm{CH}), 7.53(\mathrm{~m}, 3 \mathrm{H}, \mathrm{CH})$, $6.90(\mathrm{~d}, J=2.2 \mathrm{~Hz}, 1 \mathrm{H}, \mathrm{OCH}=\mathrm{C} \underline{\mathrm{H}}), 4.10$ (s, 3H, OMe), 4.07 (s, 3H, OMe); ${ }^{13} \mathrm{C} \mathrm{NMR}(100 \mathrm{MHz}$, $\left.\mathrm{CDCl}_{3}\right): \delta(\mathrm{ppm}) 194.6(\mathrm{C}=\mathrm{O}), 153.5,153.2,150.7,144.2,143.7,134.3,133.4,132.7,130.7,128.7$, 128.6, 127.8, 127.4, 127.1, 126.8, 123.7, 112.9, 112.0, 110.6, 105.2, 62.1, 60.5; HR-ESIMS: m/z 375.1194 $[\mathrm{M}+\mathrm{H}]^{+}$calcd for $\mathrm{C}_{23} \mathrm{H}_{18} \mathrm{O}_{5}+\mathrm{H}^{+}(375.1127) .{ }^{26}$

3-(2-Fluorophenyl)-1-(6-hydroxy-4,7-dimethoxybenzofuran-5-yl)prop-2-en-1-one （3u): orange crystals; HPLC: $t_{\mathrm{R}}=5.0$ min (95\% purity); yield: 52\%; m.p. 139-141 ${ }^{\circ} \mathrm{C}$; IR $\left(\mathrm{CHCl}_{3}\right): v_{\max } 3400,2922$, 2851, 1628, 1601, 1556, 1544, 1510, 1461, 1443, 1413, 1360, 1297,1299, 1266, $1151 \mathrm{~cm}^{-1} ;{ }^{1} \mathrm{H}$ NMR (400 MHz, $\left.\mathrm{CDCl}_{3}\right): \delta(\mathrm{ppm}) 7.96(\mathrm{q}, J=15.8 \mathrm{~Hz}, 2 \mathrm{H}, \mathrm{CH}), 7.64(\mathrm{t}, J=7.1 \mathrm{~Hz}, 1 \mathrm{H}, \mathrm{CH}), 7.53(\mathrm{~d}, J=$ $2.2 \mathrm{~Hz}, 1 \mathrm{H}, \mathrm{OC} \underline{\mathrm{H}}=\mathrm{CH},), 7.38(\mathrm{~m}, 1 \mathrm{H}, \mathrm{CH}), 7.17(\mathrm{~m}, 2 \mathrm{H}, \mathrm{CH}), 6.89(\mathrm{~d}, J=4.0 \mathrm{~Hz}, 1 \mathrm{H}, \mathrm{OCH}=\mathrm{C} \underline{\mathrm{H}}) ;{ }^{13} \mathrm{C}$ 
NMR (100 MHz, $\left.\mathrm{CDCl}_{3}\right): \delta(\mathrm{ppm}) 194.8(\mathrm{C}=\mathrm{O}), 162.8\left(\mathrm{~d},{ }^{1} J_{C F}=246 \mathrm{~Hz}\right), 160.7,153.3,152.1,150.9$, 144.1, 136.0, $131.8\left(\mathrm{~d},{ }^{3} J_{\mathrm{CF}}=8.75 \mathrm{~Hz}\right), 129.8\left(\mathrm{~d},{ }^{4} J_{\mathrm{CF}}=3.7 \mathrm{~Hz}\right), 129.6\left(\mathrm{~d},{ }^{3} J_{\mathrm{CF}}=6.0 \mathrm{~Hz}\right), 124.5\left(\mathrm{~d},{ }^{4} J_{\mathrm{CF}}\right.$ $=3.7 \mathrm{~Hz}), 123.3,116.4\left(\mathrm{~d},{ }^{2} J_{C F}=21.2 \mathrm{~Hz}\right), 112.5,111.7,105.3,61.8,61.0 ;$ HR-ESIMS: $\mathrm{m} / \mathrm{z} 432.0300$ $[\mathrm{M}+\mathrm{H}]^{+}$calcd for $\mathrm{C}_{20} \mathrm{H}_{17} \mathrm{BrO}_{6}+\mathrm{H}^{+}(432.0281) .{ }^{26}$

3-(Anthracen-2-yl)-1-(6-hydroxy-4,7-dimethoxybenzofuran-5-yl)prop-2-en-1-one $\quad(3 \mathbf{v}): \quad$ orange crystals; HPLC: $t_{\mathrm{R}}=7.4$ min (100\% purity); yield: 52\%; m.p. $156-158{ }^{\circ} \mathrm{C} ; \quad \mathrm{IR}\left(\mathrm{CHCl}_{3}\right): v_{\max } 3419$, 2920, 2850, 2103, 1632, 1569, 1442, 1408, 1359, 1215, 1146, $1065 \mathrm{~cm}^{-1} ;{ }^{1} \mathrm{H}$ NMR (400 MHz, $\left.\mathrm{CDCl}_{3}\right)$ : $\delta(\mathrm{ppm}) 8.82$ (d, $J=15.8 \mathrm{~Hz}, 1 \mathrm{H}, \mathrm{CH}), 8.49$ (s, 1H, CH), 8.40 (d, $J=8.4 \mathrm{~Hz}, 2 \mathrm{H}, \mathrm{CH}), 8.05$ (d, $J=7.4$ Hz, 2H, CH), 7.76 (d, $J=15.8 \mathrm{~Hz}, 1 \mathrm{H}, \mathrm{CH}), 7.53$ (m, 5H, CH), 6.87 (d, $J=2.2 \mathrm{~Hz}, 1 \mathrm{H}, \mathrm{OCH}=\mathrm{C} \underline{\mathrm{H}})$, 4.11 (s, 3H, OMe), 4.07 (s, 3H, OMe); $\left.{ }^{13} \mathrm{C} \mathrm{NMR} \mathrm{(100} \mathrm{MHz,} \mathrm{CDCl}\right): \delta$ (ppm) 194.3 (C=O), 153.3, 152.3, 150.9, 144.0, 140.2, 135.9, 131.3, 130.3, 129.7, 129.2, 128.9, 128.9, 128.4, 128.4, 128.3, 126.3, 126.3, 125.4, 126.4, 111.7, 111.5, 105.5, 61.4, 61.1; HR-ESIMS: $m / z$ 425.1374 [M+H] ${ }^{+}$calcd for $\mathrm{C}_{27} \mathrm{H}_{20} \mathrm{O}_{5}+\mathrm{H}^{+}$(425.1383).

Synthesis of furanoflavonones 4a-p. Khellinone (100 mg, $0.423 \mathrm{mmol}$ ) was treated with different aldehydes (1.2 equiv.) in the presence of catalytic amount of piperidine in ethanol (2 ml) at $70{ }^{\circ} \mathrm{C}$ for 12 hrs. The solvent was evaporated on rotary evaporator and the remaining mixture was diluted with water and dichloromethane. The organic layer was separated, dried over anhydrous sodium sulphate and concentrated to dryness on rotary evaporator. The obtained crude product was purified using silica gel column chromatography (5-40\%) to yield furanoflavonones 4a-p in 51-68\% yield.

4,9-Dimethoxy-7-phenyl-6,7-dihydro-5H-furo[3,2-g]chromen-5-one (4a): yellow powder; HPLC: $t_{\mathrm{R}}$ $=4.1 \min \left(100 \%\right.$ purity); yield: 65\%; m.p. $148-150{ }^{\circ} \mathrm{C} ; \mathrm{IR}\left(\mathrm{CHCl}_{3}\right): v_{\max } 3444,3021,2932,2851,2347$, 2072, 1682, 1614, 1542, 1477, 1435, 1351, 1278, 1163, 1066, $1029 \mathrm{~cm}^{-1} ;{ }^{1} \mathrm{H}$ NMR (400 MHz, $\left.\mathrm{CDCl}_{3}\right)$ : $\delta(\mathrm{ppm}) 7.52(\mathrm{~d}, J=2.2 \mathrm{~Hz}, 1 \mathrm{H}, \mathrm{OC} \underline{\mathrm{H}}=\mathrm{CH}), 7.50(\mathrm{~m}, 2 \mathrm{H}, \mathrm{CH}), 6.93(\mathrm{~d}, J=2.2 \mathrm{~Hz}, 1 \mathrm{H}, \mathrm{OCH}=\mathrm{C} \underline{\mathrm{H}})$, 5.49 (dd, $J=12.3,3.0 \mathrm{~Hz}, 1 \mathrm{H}, \mathrm{CH}), 4.08$ (s, 3H, OMe), 4.06 (s, 3H, OMe), 3.09 (dd, J = 16.7, 12.4 Hz, 1H), 2.94 (dd, $J=16.7,3.1 \mathrm{~Hz}, 1 \mathrm{H}$ ); ${ }^{13} \mathrm{C}$ NMR (100 MHz, $\left.\mathrm{CDCl}_{3}\right): \delta(\mathrm{ppm}) 190.7$ (C=O), 152.0 (C-9a), 
151.4 (C-4), 150.0 (C-8a), 144.4 (Oㅡㅐ=CH), 138.8 (C-1'), 130.0 (C-9), 128.8 (Ar-3', Ar-5'), 128.5 (Ar4), 126.0 (Ar-2', Ar-6'), 115.6 (C-4a), 111.4 (C-3a), 105.6 (OCH=ㅡㅐ), 79.3 (C-7), 61.5 (OMe), 61.4 (OMe), 46.1 (C-6); HR-ESIMS: m/z 325.1072 [M+H] ${ }^{+}$calcd for $\mathrm{C}_{19} \mathrm{H}_{16} \mathrm{O}_{5}+\mathrm{H}^{+}(325.1070)$.

7-(2-Bromophenyl)-4,9-dimethoxy-6,7-dihydro-5H-furo[3,2-g]chromen-5-one (4b): yellow powder; HPLC: $t_{\mathrm{R}}=4.3 \min \left(100 \%\right.$ purity); yield: $63 \%$; m.p. $146-148{ }^{\circ} \mathrm{C}$; IR $\left(\mathrm{CHCl}_{3}\right): v_{\max } 3708,3418,2922$, 2850, 2400, 2095, 1659, 1644, 1477, 1435, 1349, $1302 \mathrm{~cm}^{-1} ;{ }^{1} \mathrm{H}$ NMR (400 MHz, $\left.\mathrm{CDCl}_{3}\right): \delta$ (ppm) 7.68 (d, $J=1.4 \mathrm{~Hz}, 1 \mathrm{H}, \mathrm{CH}), 7.53$ (d, $J=2.3 \mathrm{~Hz}, 1 \mathrm{H}, \mathrm{OC} \underline{\mathrm{H}}=\mathrm{CH}), 7.51$ (m, 1H, CH), 7.42 (d, $J=7.8$ Hz, 1H, CH), 7.29 (d, $J=8.0 \mathrm{~Hz}, 1 \mathrm{H}, \mathrm{CH}), 6.94$ (d, $J=2.3 \mathrm{~Hz}, 1 \mathrm{H}, \mathrm{OCH}=\mathrm{C} \underline{\mathrm{H}}$ ), 5.45 (dd, $J=$ 12.2, 3.2 Hz, 1H, CH), 4.08 (s, 3H, OMe), 4.06 (s, 3H, OMe), 3.04 (dd, $J=12.2,1.3 \mathrm{~Hz}, 1 \mathrm{H}$ ), 2.94 (dd, $J=6.7$, 3.3 Hz, 1H); ${ }^{13} \mathrm{C}$ NMR (100 MHz, CDCl 3 ): $\delta$ (ppm) 190.7 (C=O), 152.0 (C-9a), 151.0 (C-4), 150.0 (C8a), 144.5 (Oㅡㅐ=CH), 141.0 (C-1'), 131.7 (C-3'), 130.4 (C-4'), 130.0 (C-9), 129.1 (C-5'), 124.6 ( C6'), 122.9 (C-2'), 115.7 (C-4a), 111.2 (C-3a), 106.7 (OCH=태), 78.4 (C-7), 61.4 (OMe), 61.4 (OMe), 46.0 (C-6); HR-ESIMS: m/z $403.0170[\mathrm{M}+\mathrm{H}]^{+}$calcd for $\mathrm{C}_{19} \mathrm{H}_{15} \mathrm{BrO}_{5}+\mathrm{H}^{+}(403.1750)$.

7-(Benzo[d][1,3]dioxol-5-yl)-4,9-dimethoxy-6,7-dihydro-5H-furo[3,2-g]chromen-5-one (4c): yellow powder; HPLC: $t_{\mathrm{R}}=3.5 \mathrm{~min}\left(100 \%\right.$ purity); yield: $60 \%$; m.p. $150-154{ }^{\circ} \mathrm{C}$; IR $\left(\mathrm{CHCl}_{3}\right): v_{\max } 3743,3584$, 3415, 2922, 2850, 1681, 1614, 1542, 1504, 1491, 1477, 1445, 1390, 1349, 1300, $1277 \mathrm{~cm}^{-1} ;{ }^{1} \mathrm{H}$ NMR (400 MHz, $\left.\mathrm{CDCl}_{3}\right): \delta(\mathrm{ppm}) 7.52(\mathrm{~d}, J=2.2 \mathrm{~Hz}, 1 \mathrm{H}, \mathrm{OC} \underline{\mathrm{H}}=\mathrm{CH}), 7.02(\mathrm{~d}, J=1.4 \mathrm{~Hz}, 1 \mathrm{H}, \mathrm{CH}), 6.94$ (d, $J=2.3 \mathrm{~Hz}, 1 \mathrm{H}, \mathrm{OCH}=\mathrm{C} \underline{\mathrm{H}}), 5.99\left(\mathrm{~s}, 2 \mathrm{H}, \mathrm{CH}_{2}\right), 5.39$ (dd, $\left.J=12.2,3.2 \mathrm{~Hz}, 1 \mathrm{H}, \mathrm{CH}\right), 4.07(\mathrm{~s}, 3 \mathrm{H}$, OMe), 4.04 (s, 3H, OMe), 3.06 (dd, $J=16.7,12.3 \mathrm{~Hz}, 1 \mathrm{H}$ ), 2.89 (dd, $J=16.7,3.1 \mathrm{~Hz}, 1 \mathrm{H}) ;{ }^{13} \mathrm{C}$ NMR (100 MHz, CDCl $): \delta$ (ppm) 190.7 (C=O), 152.0 (C-9a), 151.3 (C-4), 150.0 (C-8a), 148.0 (C-3'), 147.8 (C-4'), 144.4 (OCLH=CH), 132.6 (C-9), 129.9 (C-1'), 119.9 (C-6'), 115.5 (C-4a), 111.2 (C-3a), 108.3 (C5'), 106 (C-2'), 105.6 (OCH=대), $101.3\left(\mathrm{CH}_{2}\right), 79.2$ (C-7), 61.5 (OMe), 61.4 (OMe), 46.1 (C-6); HRESIMS: $\mathrm{m} / \mathrm{z} 369.0968[\mathrm{M}+\mathrm{H}]^{+}$calcd for $\mathrm{C}_{20} \mathrm{H}_{16} \mathrm{O}_{7}+\mathrm{H}^{+}(369.0968)$.

7-(5-Bromo-2-methoxyphenyl)-4,9-dimethoxy-6,7-dihydro-5H-furo[3,2-g]chromen-5-one (4d): yellow powder; HPLC: $t_{\mathrm{R}}=4.4 \mathrm{~min}\left(100 \%\right.$ purity); yield: $66 \%$; m.p. $178-180{ }^{\circ} \mathrm{C}$; IR $\left(\mathrm{CHCl}_{3}\right): v_{\max }$ 
3743, 3584, 3415, 2922, 2850, 1681, 1614, 1542, 1504, 1491, 1477, 1445, 1390, 1349, 1300, $1277 \mathrm{~cm}^{-1}$; ${ }^{1} \mathrm{H}$ NMR (400 MHz, $\left.\mathrm{CDCl}_{3}\right): \delta(\mathrm{ppm}) 7.79(\mathrm{~d}, J=2.0 \mathrm{~Hz}, 1 \mathrm{H}, \mathrm{CH}), 7.53(\mathrm{~d}, J=2.3 \mathrm{~Hz}, 1 \mathrm{H}, \mathrm{OC} \underline{\mathrm{H}}=\mathrm{CH})$, 7.43 (dd, $J=8.7,2.5 \mathrm{~Hz}, 1 \mathrm{H}, \mathrm{CH}), 6.94$ (d, $J=2.3 \mathrm{~Hz}, 1 \mathrm{H}, \mathrm{OCH}=\mathrm{CH}), 6.79$ (d, $J=8.8 \mathrm{~Hz}, 1 \mathrm{H}, \mathrm{CH}$ ), 5.73 (dd, $J=12.9,2.7$ Hz, 1H, CH), 4.09 (s, 3H, OMe), 4.07 (s, 3H, OMe), 3.82 (OMe), 2.99 (dd, $J=$ 16.8, $2.9 \mathrm{~Hz}, 1 \mathrm{H}), 2.83$ (dd, $J=16.8,12.9 \mathrm{~Hz}, 1 \mathrm{H}) ;{ }^{13} \mathrm{C} \mathrm{NMR}\left(100 \mathrm{MHz}, \mathrm{CDCl}_{3}\right): \delta(\mathrm{ppm}) 190.8$ (C=O), 154.8 (C-2'), 151.9 (C-9a), 151.6 (C-4), 150.1 (C-8a), 144.4 (OCLH=CH), 131.8 (C-4'), 130.1 (C-9), 129.8 (C-1'), 129.6 (C-6'), 115.8 (C-4a), 113.3 (C-3a), 112.2 (C-3'), 111.4 (C-5'), 105.6 $(\mathrm{OCH}=\underline{\mathrm{CH}}), 74.2$ (C-7), 61.5 (OMe), 61.4 (OMe), 45.1 (C-6); HR-ESIMS: m/z 433.0274 [M+H] ${ }^{+}$calcd for $\mathrm{C}_{20} \mathrm{H}_{17} \mathrm{O}_{7}+\mathrm{H}^{+}(433.0281)$.

4,9-Dimethoxy-7-(pyridin-3-yl)-6,7-dihydro-5H-furo[3,2-g]chromen-5-one (4e): yellow powder; HPLC: $t_{\mathrm{R}}=3.3$ min (100\% purity); yield: 65\%; m.p. $149-151{ }^{\circ} \mathrm{C}$; IR $\left(\mathrm{CHCl}_{3}\right): v_{\max } \quad 3584,3380,3124$, 3074, 2937, 2850, 1668, 1599, 1479, 1547, 1437, 1344, 1392, 1285, $1078 \mathrm{~cm}^{-1}$; ${ }^{1} \mathrm{H}$ NMR (400 MHz, $\left.\mathrm{CDCl}_{3}\right): \delta(\mathrm{ppm}) 8.78$ (s, 1H, CH), 8.65 (d, $\left.J=4.6 \mathrm{~Hz}, 1 \mathrm{H}, \mathrm{CH}\right), 7.86$ (d, $\left.J=7.9 \mathrm{~Hz}, 1 \mathrm{H}, \mathrm{CH}\right), 7.54$ (d, $J=2.1 \mathrm{~Hz}, 1 \mathrm{H}, \mathrm{OC} \underline{\mathrm{H}}=\mathrm{CH}), 7.38(\mathrm{dd}, J=7.8,4.8 \mathrm{~Hz}, 1 \mathrm{H}, \mathrm{CH}), 6.95(\mathrm{~d}, J=2.1 \mathrm{~Hz}, 1 \mathrm{H}, \mathrm{OCH}=\mathrm{C} \underline{\mathrm{H}})$, 5.54 (dd, $J=12.3,3.1 \mathrm{~Hz}, 1 \mathrm{H}, \mathrm{CH}), 4.09$ (s, 3H, OMe), 4.06 (s, 3H, OMe), 3.10 (dd, $J=16.6,12.4 \mathrm{~Hz}$, 1H), 2.97 (dd, $J=16.7,3.2 \mathrm{~Hz}, 1 \mathrm{H}) ;{ }^{13} \mathrm{C}$ NMR (100 MHz, $\left.\mathrm{CDCl}_{3}\right): \delta(\mathrm{ppm}) 190.8(\mathrm{C}=\mathrm{O}), 152.0$ (C-9a), 150.9 (C-4), 150.1 (C-8a), 150.0 (C-6'), 147.8 (C-4'), 144.5 (OㅡH=CH), 134.3 (C-9), 133.6 (C-2'), 130.0 (C-1'), 123.6 (C-3'), 115.8 (C-4a), 111.2 (C-3a), 106.6 (OCH=ㅡㅐ), 77.3 (C-7), 61.4 (OMe), 61.3 (OMe), 45.6 (C-6); HR-ESIMS: m/z 326.1021 [M+H] $]^{+}$calcd for $\mathrm{C}_{18} \mathrm{H}_{16} \mathrm{NO}_{5}+\mathrm{H}^{+}$(325.1023). 4,9-Dimethoxy-7-(thiophen-3-yl)-6,7-dihydro-5H-furo[3,2-g]chromen-5-one (4f): yellow powder; HPLC: $t_{\mathrm{R}}=5.3 \min \left(89 \%\right.$ purity); yield: 66\%; m.p. $159-161{ }^{\circ} \mathrm{C}$; IR $\left(\mathrm{CHCl}_{3}\right): v_{\max } 3428,1927,2851$, 2079, 1673, 1651, 1620, 1477, 1542, 1435, 1348, 1300, $1216 \mathrm{~cm}^{-1} ;{ }^{1} \mathrm{H} \mathrm{NMR}\left(400 \mathrm{MHz}, \mathrm{CDCl}_{3}\right): \delta$ (ppm) 7.52 (d, $J=2.2 \mathrm{~Hz}, 1 \mathrm{H}, \mathrm{OC} \underline{\mathrm{H}}=\mathrm{CH}), 7.37$ (m, 2H, CH), 7.21 (dd, $J=4.9,1.3 \mathrm{~Hz}, 1 \mathrm{H}, \mathrm{CH}), 6.92$ (d, $J=2.3 \mathrm{~Hz}, 1 \mathrm{H}, \mathrm{OCH}=\mathrm{C} \underline{\mathrm{H}}), 5.60$ (dd, $J=10.3,4.1 \mathrm{~Hz}, 1 \mathrm{H}, \mathrm{CH}), 4.07$ (s, 3H, OMe), 4.06 (s, 3H, OMe), 3.12 (dd, $J=16.7,10.3 \mathrm{~Hz}, 1 \mathrm{H}), 3.04$ (dd, $J=16.7,4.1 \mathrm{~Hz}, 1 \mathrm{H}) ;{ }^{13} \mathrm{C}$ NMR (100 MHz, $\left.\mathrm{CDCl}_{3}\right)$ : 
$\delta(\mathrm{ppm}) 190.5$ (C=O), 152.0 (C-9a), 151.0 (C-4), 150.0 (C-8a), 144.4 (OㄷH=CH), 139.8 (C-1'), 128.8 (C-9), 126.7 (C-3'), 125.8 (C-2'), 122.6 (C-5'), 115.5 (C-4a), 111.4 (C-3a), 106.7 (OCH=ㅡㅐ), 75.4 (C7), 61.4 (OMe), 61.4 (OMe), 45.1 (C-6); HR-ESIMS: m/z $331.0643[\mathrm{M}+\mathrm{H}]^{+}$calcd for $\mathrm{C}_{17} \mathrm{H}_{14} \mathrm{O}_{5} \mathrm{~S}+\mathrm{H}^{+}$ (331.0634).

4,9-Dimethoxy-7-(4-nitrophenyl)-6,7-dihydro-5H-furo[3,2-g]chromen-5-one (4g): yellow powder; HPLC: $t_{\mathrm{R}}=3.6$ min $\left(100 \%\right.$ purity); yield: 61\%; m.p. $180-181^{\circ} \mathrm{C}$; IR $\left(\mathrm{CHCl}_{3}\right): v_{\max } 3745,3383,2850$, 2918, 1734, 1702, 1604, 1519, 1503, 1467, 1345, 1273, 1213, 1149, $1066 \mathrm{~cm}^{-1} ;{ }^{1} \mathrm{H}$ NMR (400 MHz, $\left.\mathrm{CDCl}_{3}\right): \delta(\mathrm{ppm}) 8.10(\mathrm{~d}, J=8.7 \mathrm{~Hz}, 2 \mathrm{H}, \mathrm{CH}), 7.56-7.49(\mathrm{dd}, J=7.6,5.5 \mathrm{~Hz}, 2 \mathrm{H}, \mathrm{CH}), 6.91(\mathrm{~d}, J=2.3$ Hz, 1H, OCH=C프), 4.88 (dd, $J=6.5,4.4 \mathrm{~Hz}, 1 \mathrm{H}, \mathrm{CH}), 4.12$ (s, 3H, OMe), 4.06 (s, 3H, OMe), 3.47 (dd, $J=16.2,4.0 \mathrm{~Hz}, 1 \mathrm{H}), 3.04$ (dd, $J=14.6,6.6 \mathrm{~Hz}, 1 \mathrm{H}) ;{ }^{13} \mathrm{C}$ NMR (100 MHz, CDCl $): \delta(\mathrm{ppm}) 196.5$ (C=O), 159.6 (C-9a), 154.7 (C-4), 147.1 (C-1'), 146.9 (C-4'), 144.5 (OCLH=CH), 143.1 (C-8a), 130.6 (C2', C-6'), 127.7 (C-9), 123.5 (C-3', C-5'), 113.8 (C-4a), 108.3 (C-3a), 106.1 (OCH=ㅡㅐ), 85.4 (C-7), 61.2 (OMe), 60.8 (OMe), 37.1 (C-6); HR-ESIMS: m/z 370.0933 [M+H] $]^{+}$calcd for $\mathrm{C}_{19} \mathrm{H}_{15} \mathrm{NO}_{7}+\mathrm{H}^{+}$ (370.0931).

7-(2-Chlorophenyl)-4,9-dimethoxy-6,7-dihydro-5H-furo[3,2-g]chromen-5-one (4h): yellow powder; HPLC: $t_{\mathrm{R}}=4.4$ min (100\% purity); yield: 65\%; m.p. $142-143{ }^{\circ} \mathrm{C} ; \mathrm{IR}\left(\mathrm{CHCl}_{3}\right): v_{\max } 3625,3584,3441$, 2934, 2849, 2079, 1682, 1651, 1542, 1435, 1391, 1303, $1278 \mathrm{~cm}^{-1} ;{ }^{1} \mathrm{H}$ NMR (400 MHz, $\left.\mathrm{CDCl}_{3}\right): \delta$ (ppm) 7.67 (t, $J=7.3 \mathrm{~Hz}, 1 \mathrm{H}, \mathrm{CH}), 7.54$ (d, $J=2.1 \mathrm{~Hz}, 1 \mathrm{H}, \mathrm{OC} \underline{\mathrm{H}}=\mathrm{CH}), 7.40-7.34$ (m, 1H, CH), 7.25 (dd, $J=12.2,4.4 \mathrm{~Hz}, 1 \mathrm{H}, \mathrm{CH}), 7.15-7.08$ (m, 1H, CH), 6.95 (d, $J=2.1 \mathrm{~Hz}, 1 \mathrm{H}, \mathrm{OCH}=\mathrm{CH}), 5.77$ (dd, $J$ =12.5, 3.0 Hz, 1H), 4.12 (s, 3H, OMe), 4.06 (s, 3H, OMe), 3.07 (dd, $J=16.7,12.6 \mathrm{~Hz}, 1 \mathrm{H}$ ), 2.96 (dd, $J$ = 16.8, 3.2 Hz, 1H); ${ }^{13} \mathrm{C}$ NMR (100 MHz, $\left.\mathrm{CDCl}_{3}\right): \delta(\mathrm{ppm}) 190.3(\mathrm{C}=\mathrm{O}), 152.0$ (C-9a), 151.4 (C-4), 150.2 (C-8a), 144.5 (OㄷH=CH), 136.7 (C-1'), 131.7 (C-2'), 130.0 (C-9), 129.7 (C-3'), 129.5 (C-4'), 127.4 (C-6'), 127.1 (C-5'), 115.7 (C-4a), 111.3 (C-3a), 105.7 (OCH=ㅡㅐ), 77.5 (C-7), 61.5 (OMe), 61.5 (OMe), 44.9 (C-6); HR-ESIMS: m/z $359.0670[\mathrm{M}+\mathrm{H}]^{+}$calcd for $\mathrm{C}_{19} \mathrm{H}_{15} \mathrm{ClO}_{5}+\mathrm{H}^{+}(359.0675)$. 
(2,4-Dichlorophenyl)-4,9-dimethoxy-6,7-dihydro-5H-furo[3,2-g]chromen-5-one (4i): yellow powder;

HPLC: $t_{\mathrm{R}}=4.5 \min \left(100 \%\right.$ purity); yield: 64\%; m.p. $123-125{ }^{\circ} \mathrm{C}$; IR $\left(\mathrm{CHCl}_{3}\right): v_{\max } 3584,3354,3019$, 2930, 2850, 1683, 1614, 1543, 1479, 1485, 1348, 1278, 1165, 1130, 1102, $1018 \mathrm{~cm}^{-1} ;{ }^{1} \mathrm{H}$ NMR (400 $\left.\mathrm{MHz}, \mathrm{CDCl}_{3}\right): \delta(\mathrm{ppm}) 7.79(\mathrm{~d}, J=8.4 \mathrm{~Hz}, 1 \mathrm{H}, \mathrm{CH}), 7.55(\mathrm{~d}, J=2.1 \mathrm{~Hz}, 1 \mathrm{H}, \mathrm{OC} \underline{\mathrm{H}}=\mathrm{CH}), 7.44(\mathrm{~m}, 1 \mathrm{H}$, CH), 7.38 (dd, $J=8.4,2.1 \mathrm{~Hz}, 1 \mathrm{H}, \mathrm{CH}), 6.96$ (d, $J=2.3 \mathrm{~Hz}, 1 \mathrm{H}, \mathrm{OCH}=\mathrm{CH}), 5.78$ (dd, $J=13.0,2.8$ Hz, 1H, CH), 4.11 (s, 3H, OMe), 4.05 (s, 3H, OMe), 3.03 (dd, $J=16.8,2.9$ Hz, 1H), 2.86 (dd, $J=16.8$, $13.0 \mathrm{~Hz}, 1 \mathrm{H}) ;{ }^{13} \mathrm{C}$ NMR (100 MHz, $\left.\mathrm{CDCl}_{3}\right): \delta(\mathrm{ppm}) 189.9$ (C=O), 152.0 (C-9a), 151.1 (C-4), 150.2 (C8a), 144.6 (OㄷH=CH), 135.3 (C-9), 134.8 (C-1'), 132.3 (C-4'), 130.1 (C-2'), 129.5 (C-3'), 128.1 (C-6'), 127.8 (C-5'), 115.8 (C-4a), 111.1 (C-3a), 105.7 (OCH=대), 76.0 (C-7), 61.5 (OMe), 61.5 (OMe), 44.8 (C-6); HR-ESIMS: m/z $393.0282[\mathrm{M}+\mathrm{H}]^{+}$calcd for $\mathrm{C}_{19} \mathrm{H}_{15} \mathrm{Cl}_{2} \mathrm{O}_{5}+\mathrm{H}^{+}$(393.0291).

4,9-Dimethoxy-7-(naphthalen-2-yl)-6,7-dihydro-5H-furo[3,2-g]chromen-5-one (4j): yellow powder; HPLC: $t_{\mathrm{R}}=5.2 \min \left(100 \%\right.$ purity); yield: 60\%; m.p. $143-145{ }^{\circ} \mathrm{C}$; IR $\left(\mathrm{CHCl}_{3}\right): v_{\max } 3418,2925,2852$, 2085, 1680, 1616, 1545, 1497, 1436, 1390, 1302, 1249, 1126, $1018 \mathrm{~cm}^{-1} ;{ }^{1} \mathrm{H}$ NMR (400 MHz, $\left.\mathrm{CDCl}_{3}\right)$ : $\delta(\mathrm{ppm}) 7.79$ (m, 4H, CH), 7.63 (d, $J=8.5 \mathrm{~Hz} .1 \mathrm{H}, \mathrm{OC} \underline{\mathrm{H}}=\mathrm{CH}), 7.52(\mathrm{~m}, 3 \mathrm{H}, \mathrm{CH}), 6.94(\mathrm{~d}, J=2.2 \mathrm{~Hz}$, 1H, OCH=Cㅌ) 5.66 (dd, $J=16.7,12.2 \mathrm{~Hz}, 1 \mathrm{H}, \mathrm{CH}), 4.09$ (s, 3H, OMe), 4.07 (s, 3H, OMe), 3.20 (dd, $J=16.7,12.2 \mathrm{~Hz}, 1 \mathrm{H}), 3.04(\mathrm{dd}, J=16.7,3.1 \mathrm{~Hz}, 1 \mathrm{H}) ;{ }^{13} \mathrm{C}$ NMR $\left(100 \mathrm{MHz}, \mathrm{CDCl}_{3}\right): \delta(\mathrm{ppm}) 190.7$ (C=O), 152.1 (C-9a), 151.4 (C-4), 150.1 (C-8a), 144.4 (OㄷH=CH), 136.1 (C-9), 133.8 (C-8'), 133.1 (C3'), 130.0 (C-1'), 128.7 (C-9'), 128.2 (C-10'), 127.7 (C-4', C-7'), 115.6 (C-3a), 111.4 (C-4a), 106.7 (OCH=태), 79.4 (C-7), 61.5 (OMe), 61.4 (OMe), 46.0 (C-6), HR-ESIMS: m/z 375.1230 [M+H] $]^{+}$calcd for $\mathrm{C}_{23} \mathrm{H}_{18} \mathrm{O}_{5}+\mathrm{H}^{+}$(375.1227).

7-(2,6-Dichlorophenyl)-4,9-dimethoxy-6,7-dihydro-5H-furo[3,2-g]chromen-5-one (4k): yellow powder; HPLC: $t_{\mathrm{R}}=4.4$ min (100\% purity); yield: 65\%; m.p. $143-145{ }^{\circ} \mathrm{C}$; IR $\left(\mathrm{CHCl}_{3}\right): v_{\max } 3444$, 3011, 2936, 2848, 2111, 1682, 1614, 1581, 1564, 1477, 1434, 1376, 1348, 1277, 1247, $1211 \mathrm{~cm}^{-1} ;{ }^{1} \mathrm{H}$ NMR (400 MHz, $\left.\mathrm{CDCl}_{3}\right): \delta(\mathrm{ppm}) 7.53$ (d, $\left.J=2.2 \mathrm{~Hz}, 1 \mathrm{H}, \mathrm{OC} \underline{\mathrm{H}}=\mathrm{CH}\right), 7.39$ (d, $\left.J=8.0 \mathrm{~Hz}, 2 \mathrm{H}, \mathrm{CH}\right)$, 7.28 (m, 1H, CH), 6.95 (d, $J=2.2 \mathrm{~Hz}, 1 \mathrm{H}, \mathrm{OCH}=\mathrm{CH}), 6.25$ ( dd, $J=14.4,3.1 \mathrm{~Hz}, 1 \mathrm{H}, \mathrm{CH}), 4.11$ (s, 
3H, OMe), 4.03 (s, 3H, OMe), 3.77 (dd, $J=16.8,14.5 \mathrm{~Hz}, 1 \mathrm{H}), 2.69$ (dd, $J=16.8,3.1 \mathrm{~Hz}, 1 \mathrm{H}) ;{ }^{13} \mathrm{C}$ NMR (100 MHz, CDCl $): \delta$ (ppm) 190.1 (C=O), 152.3 (C-9a), 151.5 (C-4), 150.3 (C-8a), 144.5 (OCEH=CH), 135.1 (C-9), 134.8 (C-1'),132.5 (C-2', C-6'), 130.2 (C-3'), 129.7 (C-4'), 128.9 (C-5'), 115.4 (C-3a), 111.0 (C-4a), 105.7 (OCH=CHH), 75.9 (C-7), 61.9 (OMe), 61.5 (OMe), 41.0 (C-6); HR-ESIMS: $m / z$ 393.0331 $[\mathrm{M}+\mathrm{H}]^{+}$calcd for $\mathrm{C}_{19} \mathrm{H}_{14} \mathrm{Cl}_{2} \mathrm{O}_{5}+\mathrm{H}^{+}$(393.0291).

7-(3-Bromo-4-fluorophenyl)-4,9-dimethoxy-6,7-dihydro-5H-furo[3,2-g]chromen-5-one (4l): yellow powder; HPLC: $t_{\mathrm{R}}=4.1 \mathrm{~min}$ (95\% purity); yield: 68\%; m.p. $140-143{ }^{\circ} \mathrm{C}$; IR $\left(\mathrm{CHCl}_{3}\right): v_{\max } 3429,2933$, 2850, 2091, 1681, 1614, 1544, 1498, 1478, 1435, 1417, 1391, 1349, 1278, 1249, $1067 \mathrm{~cm}^{-1} ;{ }^{1} \mathrm{H}$ NMR (400 MHz, $\left.\mathrm{CDCl}_{3}\right): \delta(\mathrm{ppm}) 7.73$ (dd, $\left.J=6.4,2.0 \mathrm{~Hz}, 1 \mathrm{H}, \mathrm{CH}\right), 7.54(\mathrm{~d}, J=2.2 \mathrm{~Hz}, 1 \mathrm{H}, \mathrm{OC} \underline{\mathrm{H}}=\mathrm{CH})$, 7.44-7.39 (m, 1H, CH), 7.18 (t, $J=8.4 \mathrm{~Hz}, 1 \mathrm{H}, \mathrm{CH}), 6.95$ (d, $J=2.2 \mathrm{~Hz}, 1 \mathrm{H}, \mathrm{OCH}=\mathrm{C} \underline{\mathrm{H}}), 5.43$ (dd, $J=$ 12.1, 3.1 Hz, 1H, CH), 4.08 (s, 3H, OMe), 4.06 (s, 3H, OMe), 3.03 (dd, J = 16.7, $12.1 \mathrm{~Hz}, 1 \mathrm{H}), 2.92$ (dd, $J=16.7,3.3 \mathrm{~Hz}, 1 \mathrm{H}) ;{ }^{13} \mathrm{C} \mathrm{NMR}\left(125 \mathrm{MHz}, \mathrm{CDCl}_{3}\right): \delta(\mathrm{ppm}) 189.9(\mathrm{C}=\mathrm{O}), 160.0\left(\mathrm{~d},{ }^{1} J_{C F}=246\right.$ Hz, C-4'), 158.0 (C-9a), 152.4 (C-4), 150.8 (C-8a), 150.1 (C-9) 144.6 (OㄷH=CH), 136.2 (d, ${ }^{4} J_{C F}=3.7$ Hz, CH-1'), 131.4 (C-2'), 129.9 (C-3a), 126.8 (d, ${ }^{3} J_{C F}=7.2$ Hz, CH-6'), 116.9 (CH-5'), 111.1 (C-4a), $109.6\left(\mathrm{~d},{ }^{2} J_{C F}=21.2 \mathrm{~Hz}, \mathrm{C}-3\right.$ '), $106.7(\mathrm{OCH}=\underline{\mathrm{CH}}), 77.9$ (C-7), 61.4 (OMe), 61.4 (OMe), 45.9 (C-6); HR-ESIMS: $m / z$ 423.0060 $[\mathrm{M}+\mathrm{H}]^{+}$calcd for $\mathrm{C}_{19} \mathrm{H}_{14} \mathrm{BrFO}_{5}+\mathrm{H}^{+}(423.0060)$.

7-(2-Fluorophenyl)-4,9-dimethoxy-6,7-dihydro-5H-furo[3,2-g]chromen-5-one (4m): yellow powder; HPLC: $t_{\mathrm{R}}=3.7 \min \left(93 \%\right.$ purity); yield: 64\%; m.p. $142-145{ }^{\circ} \mathrm{C}$; IR $\left(\mathrm{CHCl}_{3}\right): v_{\max } 3584,3360,3126$, 3070, 2938, 2843, 1687, 1682, 1615, 1543, 1505, 1478, 1459, 1436, 1349, 1302, 1278, 1252, 1233, 1165, 1130, $1067 \mathrm{~cm}^{-1}$; ${ }^{1} \mathrm{H}$ NMR (400 MHz, $\left.\mathrm{CDCl}_{3}\right): \delta(\mathrm{ppm}) 7.67(\mathrm{t}, J=7.3 \mathrm{~Hz}, 1 \mathrm{H}, \mathrm{CH}), 7.54(\mathrm{~d}, J=$ $2.1 \mathrm{~Hz}, 1 \mathrm{H}, \mathrm{OC} \underline{\mathrm{H}}=\mathrm{CH}), 7.37$ (dd, $J=13.3,6.0 \mathrm{~Hz}, 1 \mathrm{H}, \mathrm{CH}), 7.25$ (dd, 1H, $J=12.2,4.4 \mathrm{~Hz}, \mathrm{CH}), 7.15-$ $7.08(\mathrm{~m}, 1 \mathrm{H}, \mathrm{CH}), 6.95(\mathrm{~d}, J=2.1 \mathrm{~Hz}, 1 \mathrm{H}, \mathrm{OCH}=\mathrm{CH}), 5.77(\mathrm{dd}, J=12.5,3.0 \mathrm{~Hz}, 1 \mathrm{H}, \mathrm{CH}), 4.10$ (s, 3H, OMe), 4.05 (s, 3H, OMe), 3.07 (dd, $J=16.7,12.6 \mathrm{~Hz}, 1 \mathrm{H}), 2.96$ (dd, $J=16.8,3.2 \mathrm{~Hz}, 1 \mathrm{H}) ;{ }^{13} \mathrm{C}$ NMR (125 MHz, $\left.\mathrm{CDCl}_{3}\right): \delta(\mathrm{ppm}) 190.4(\mathrm{C}=\mathrm{O}), 160.7$ (d, ${ }^{1} J_{\mathrm{CF}}=246 \mathrm{~Hz}, \mathrm{C}-2$ '), 158.7 (C-9a), 152.1 (C4), 151.4 (C-8a), 150.2 (C-9), 144.5 (OㄷH=CH), 130.2 (d, ${ }^{3} J_{C F}=8.7$ Hz, CH-4'), 130.0 (C-3a), 127.4 
(CH-6'), $126.2\left(\mathrm{CH}-1^{\prime}\right), 124.6$ (d, ${ }^{4} J_{C F}=3.7 \mathrm{~Hz}$ CH-5'), 115.8 (d, ${ }^{2} J_{C F}=21.2 \mathrm{~Hz}, \mathrm{CH}-3$ '), $111.1(\mathrm{C}-$ 4a), 105.7 (OCH=ㅌH), 73.8 (CH-7), 61.5 (OMe), 61.5 (OMe), 45.1 (C-6); HR-ESIMS: m/z 343.0990 $[\mathrm{M}+\mathrm{H}]^{+}$calcd for $\mathrm{C}_{19} \mathrm{H}_{15} \mathrm{FO}_{5}+\mathrm{H}^{+}(343.0976)$.

7-(4-Fluorophenyl)-4,9-dimethoxy-6,7-dihydro-5H-furo[3,2-g]chromen-5-one (4n): yellow powder; HPLC: $t_{\mathrm{R}}=4.4 \min \left(100 \%\right.$ purity); yield: 61\%; m.p. $142-145^{\circ} \mathrm{C} ; \mathrm{IR}\left(\mathrm{CHCl}_{3}\right): v_{\max } 3744,3397,2920$, 2351, 1683, 1575, 1512, 1477, 1414, 1348, 1301, 1226, 1126, $1065 \mathrm{~cm}^{-1} ;{ }^{1} \mathrm{HNMR}\left(400 \mathrm{MHz}, \mathrm{CDCl}_{3}\right): \delta$ (ppm) 7.53 (d, $J=2.2 \mathrm{~Hz}, 1 \mathrm{H}, \mathrm{OC} \underline{\mathrm{H}}=\mathrm{CH}), 7.49$ (dd, $J=8.5,5.4 \mathrm{~Hz}, 2 \mathrm{H}, \mathrm{CH}), 7.12(\mathrm{t}, J=8.6 \mathrm{~Hz}, 1 \mathrm{H}$, CH), 6.94 (d, $J=2.2 \mathrm{~Hz}, 1 \mathrm{H}, \mathrm{OCH}=\mathrm{CH}), 5.47$ (dd, $J=12.3,2.9 \mathrm{~Hz}, 1 \mathrm{H}, \mathrm{CH}), 4.09$ (s, 3H, OMe), 4.05 (s, 3H, OMe), 3.07 (dd, $J=16.7,12.3 \mathrm{~Hz}, 1 \mathrm{H}), 2.93$ (dd, $J=16.7,3.2 \mathrm{~Hz}, 1 \mathrm{H}$ ); ${ }^{13} \mathrm{C}$ NMR $(125 \mathrm{MHz}$ $\left.\mathrm{CDCl}_{3}\right): \delta(\mathrm{ppm}) 190.5(\mathrm{C}=\mathrm{O}), 163.7$ (d, ${ }^{1} J_{C F}=246 \mathrm{~Hz}, \mathrm{C}-4$ '), 161.7 (C-9a), 152.0 (C-4), 151.2 (C-8a), 150.0 (C-9), 144.5 (OㄷH=CH), $134.6\left(\mathrm{CH}-1^{\prime}\right), 127.9$ (d, ${ }^{3} J_{C F}=8.7 \mathrm{~Hz}, \mathrm{C}-2$ ', C-6'), $115.8\left(\mathrm{~d},{ }^{2} J_{C F}=20.0\right.$ Hz, C-3', C-5'), 115.6 (C-3a), 111.2 (C-4a), 105.6 (OCH=ㅡㅐ), 78.6 (C-7), 61.4 (OMe), 61.4 (OMe), 45.9 (C-6); HR-ESIMS: m/z $343.0990[\mathrm{M}+\mathrm{H}]^{+}$calcd for $\mathrm{C}_{19} \mathrm{H}_{15} \mathrm{FO}_{5}+\mathrm{H}^{+}$(343.0976).

(E)-6-Benzylidene-4,9-dimethoxy-7-phenyl-6,7-dihydro-5H-furo[3,2-g]chromen-5-one (4o): yellow powder; HPLC: $t_{\mathrm{R}}=3.7 \mathrm{~min}$ (87\% purity); yield: 51\%; m.p. $159-161^{\circ} \mathrm{C} ; \mathrm{IR}\left(\mathrm{CHCl}_{3}\right): v_{\max } 3585,3415$, 2922, 2850, 2094, 1641, 1619, 1475, 1437, 1348, 1277, 1126, $\mathrm{cm}^{-1} ;{ }^{1} \mathrm{H}$ NMR (400 MHz, $\left.\mathrm{CDCl}_{3}\right): \delta$ (ppm) 8.09 (s, 1H, CH), 7.98 (d, J = $2.1 \mathrm{~Hz}, 1 \mathrm{H}, \mathrm{CH}), 7.50$ (m, 5H, CH), 7.44 (t, J = 6.9 Hz, 4H), 7.38 (d, $J=7.1 \mathrm{~Hz}, 1 \mathrm{H}, \mathrm{CH}), 7.18$ (d, $J=2.1 \mathrm{~Hz}, 1 \mathrm{H}, \mathrm{CH}), 6.76$ (s, 1H, CH), 4.00 (s, 3H, OMe), 3.94 (s, 3H, OMe); ${ }^{13} \mathrm{C}$ NMR (100 MHz, $\left.\mathrm{CDCl}_{3}\right): \delta(\mathrm{ppm})$ 180.0, 151.2, 149.8, 147.6, 145.4, 138.0, 137.1, 133.3, 132.5, 130.1, 129.9, 129.8, 129.0, 128.9, 127.2, 115.3, 111.7, 105.7, 77.2, 61.1, 60.8; HR-ESIMS: m/z 413.1383 $[\mathrm{M}+\mathrm{H}]^{+}$calcd for $\mathrm{C}_{26} \mathrm{H}_{20} \mathrm{O}_{5}+\mathrm{H}^{+}$(413.1383).

\section{(Z)-4,9-Dimethoxy-6-(thiophen-2-ylmethylene)-7-(thiophen-3-yl)-6,7-dihydro-5H-furo[3,2-}

g]chromen-5-one (4p): yellow powder; HPLC: $t_{\mathrm{R}}=4.6$ min (95\% purity); yield: 55\%; m.p. 148-150 ${ }^{\circ} \mathrm{C}$; IR $\left(\mathrm{CHCl}_{3}\right): v_{\max }$ 3429, 2933, 2850, 2091, 1681, 1614, 1544, 1498, 1478, 1435, 1417, 1391, 1349, 1278, 1249, $1067 \mathrm{~cm}^{-1} ;{ }^{1} \mathrm{H}$ NMR (400 MHz, $\left.\mathrm{CDCl}_{3}\right): \delta$ (ppm) 7.94-7.91 (m, 2H, CH), 7.86 (d, $J=1.9$ 
Hz, 1H, CH), 7.67 (dd, $J=4.9,2.9 \mathrm{~Hz}, 1 \mathrm{H}, \mathrm{CH}), 7.53$ (d, $J=2.9 \mathrm{~Hz}, 1 \mathrm{H}, \mathrm{OC} \underline{\mathrm{H}}=\mathrm{CH}), 7.38-7.34$ (m,

1H, CH), 7.24 (d, $J=5.0 \mathrm{~Hz}, 1 \mathrm{H}, \mathrm{CH}), 7.18$ (d, $J=5.0 \mathrm{~Hz}, 1 \mathrm{H}, \mathrm{CH}), 7.13$ (d, $J=2.2 \mathrm{~Hz}, 1 \mathrm{H}, 1 \mathrm{H}), 6.83$

(s, 1H, CH), 3.96 (s, 3H, OMe), 3.86 (s, 3H, OMe); ${ }^{13} \mathrm{C}$ NMR (100 MHz, CDCl $): \delta(p p m) 180.8$ (C=O), 151.6, 150.2, 148.4, 144.1, 139.1, 135.8, 132.1, 131.0, 130.8, 129.1, 128.6, 127.0, 126.8, 126.7, 124.7, 116.5, 112.7, 105.5, 75.1, 61.5, 61.4; HR-ESIMS: $\mathrm{m} / \mathrm{z} 425.0495[\mathrm{M}+\mathrm{H}]^{+}$calcd for $\mathrm{C}_{22} \mathrm{H}_{16} \mathrm{O}_{5} \mathrm{~S}_{2}+$ $\mathrm{H}^{+}(425.0511)$.

CYP screening and protection from B[a]P toxicity. The activity of test compounds for inhibition of CYP enzymes was investigated using Sacchrosomes ${ }^{\mathrm{TM}}$ and recombinant human HEK293 cells using protocols, as described in our earlier publications. ${ }^{10,12}$ The protection from B[a]P toxicity in CYP1A1expressing normal adherent HEK293 human cells was studied as per protocols described in our earlier publications. $^{10,12}$

For $\mathrm{IC}_{50}$ determination for CYP inhibition, the enzyme inhibition was plotted using sigmoidal curve (4 parameter variable slope equation) and half-inhibitory concentration ( $\left.\mathrm{IC}_{50}\right)$ values were analyzed statistically using GraphPad Prism Software (Version 6.0). The $\mathrm{IC}_{50}$ values of CYP inhibitors were determined by fitting a non-linear curve of uninhibited fraction with incubation concentration of the inhibitors, using the Hill equation. The $\mathrm{EC}_{50}$ values for $\mathrm{B}[\mathrm{a}] \mathrm{P}$ toxicity were determined by plotting doseresponse curve of cell viability versus log concentration of $\mathrm{B}[\mathrm{a}] \mathrm{P}$ or $\mathrm{B}[\mathrm{a}] \mathrm{P}+$ test compound. $\mathrm{EC}_{50}$ values were obtained using GraphPad Prism Software (Version 6.0), indicating the concentration of B[a]P required for $50 \%$ reduction in cell viability.

Profiling of khellinochalcones on a panel of 12 humanized CYP450 enzymes. IC 50 values of furanochalcone $\mathbf{3 g}$ were obtained by kinetic microplate assay using 15 minutes reaction time. Recombinant human P450s from a Saccharomyces cerevisiae microsome expression system supplied from CYP Design was used. Each $100 \mu \mathrm{l}$ reaction was performed in $100 \mathrm{mM}$ potassium phosphate buffer, pH 7.4 and with a NADPH regenerating system (final well concentrations 0.04 units of glucose6-phosphate dehydrogenase, $1.3 \mathrm{mM} \mathrm{NADP}^{+}$, $3.3 \mathrm{mM}$ glucose-6-phosphate, $3.3 \mathrm{mM} \mathrm{MgCl} 2$ and $50 \mu \mathrm{M}$ 
tribasic sodium citrate). Test samples were serially diluted in 10\% DMSO in decreasing final well concentrations from $20 \mu \mathrm{M}$ downwards/ $0.5 \%$ DMSO. CYP2E1 is sensitive to solvents and so test samples were diluted in 1\% DMSO in decreasing final well concentrations from $10 \mu \mathrm{M}$ downwards/ 0.5 \% DMSO. The assay was initiated by adding substrate and the NADPH regenerating system. P450 content and substrate concentrations were pre-optimised. ${ }^{12}$

In vitro cell proliferation assays and flow cytometric analyses. They were performed as described earlier. ${ }^{28}$ Control cells (i.e. in the absence of any compound) and cells (i.e. treated with compound) cells were harvested after treatment with trypsin. Cells were fixed in chilled $\left(-20^{\circ} \mathrm{C}\right) 70 \%$ ethanol for an hour, after washing once with PBS. Fixed cells were centrifuged at room temperature. Pellets were re-suspended in PBS, in the presence of DNase-free ribonuclease $(0.5 \mathrm{mg} / \mathrm{mL}$; SigmaAldrich \# R-5503) before staining with a propidium iodide solution (50 $\mu \mathrm{g} / \mathrm{mL}$; Sigma-Aldrich, \# P4170) for an hour (in the dark) at $4^{\circ} \mathrm{C}$. Cell cycle analysis was performed on the Beckman Coulter (Epics Altra) fluorescence-activated cell sorter (Beckman Coulter UK Ltd). To exclude cell doublets or cell clumps, all events that represent single cells were gated. Cytograms of the fluorescence peak of propidium iodide were plotted against the integrated fluorescence/linear signal. Data points on a straight line, within a single gate, were isolated and the gated data was employed for plotting a histogram that represents a complete cell cycle. The total number of events did not exceed 200 events per second. Data acquisition was stopped after collection of around 10,000 events.

In silico docking with CYP1A1. The docking of identified CYP1A1 inhibitors with the crystal structure of CYP1A1 (PDB ID: 4I8V) ${ }^{18}$ was performed using the protocol as described earlier. ${ }^{10,12}$

\section{AUTHOR INFORMATION.}

Corresponding Authors.

*Tel: +91 191 2569006; Fax: +91 191 2569333; E-mail: sbharate@iiim.ac.in (SBB). 
* Tel. 00-44-7805230121; E-mail: bchaud00@gmail.com (BC)

\section{ORCID}

Sandip B. Bharate: $\quad$ 0000-0001-6081-5787

Bhabatosh Chaudhuri: $\quad$ 0000-0002-0331-8634

\section{Author Contributions}

${ }^{\text {\# }}$ R.S. and I.S.W. contributed equally to this work as a first author.

\section{Author Contributions}

S.B. Bharate and B. Chaudhuri designed, executed and coordinated this whole study; R. Sharma performed synthesis of compounds; I.S. Williams, L. Gatchie and V.R. Sonawane performed all CYP screening; S.B. Bharate and B. Chaudhuri contributed to manuscript writing.

\section{Funding}

This work was supported by CSIR $12^{\text {th }}$ five year plan project (BSC-0205) and CYP Design Ltd.

Notes. The authors declare no competing financial interest.

ACKNOWLEDGEMENTS. Authors thank analytical department, IIIM for analytical support.

\section{ASSOCIATED CONTENT.}

The Supporting Information is available free of charge via the Internet at http://pubs.acs.org.

- ${ }^{1} \mathrm{H},{ }^{13} \mathrm{C}$, DEPT NMR, data scans of all compounds

\section{E-MAIL ADDRESSES OF ALL AUTHORS.}

Rajni Sharma (ranjupagotra@gmail.com), Ibidapo S. Williams (williams.i@hotmail.co.uk), Linda Gatchie (linda.gatchie@cypdesign.co.uk), Vinay R. Sonawane (vinaysonawane@rediffmail.com), Bhabatosh Chaudhuri (bchaud00@gmail.com), Sandip B. Bharate (sbharate@iiim.ac.in).

\section{REFERENCES}


(1). (a) Duarte, J.; Perez-Vizcaino, F.; Torres, A. I.; Zarzuelo, A.; Jimenez, J.; Tamargo, J. Vasodilator effects of visnagin in isolated rat vascular smooth muscle. Eur. J. Pharmacol. 1995, 286, 115-122; (b) Duarte, J.; Torres, A. I.; Zarzuelo, A. Cardiovascular effects of visnagin on rats. Planta Med. 2000, 66, 35-39; (c) Khan, Z. A.; Assiri, A. M.; Al-Afghani, H. M.; Maghrabi, T. M. Inhibition of oxalate nephrolithiasis with Ammi visnaga (AI-Khillah). Int. Urol. Nephrol. 2001, 33, 605-608; (d) de Leeuw, J.; Assen, Y. J.; van der Beek, N.; Bjerring, P.; Martino Neumann, H. A. Treatment of vitiligo with khellin liposomes, ultraviolet light and blister roof transplantation. J. Eur. Acad. Dermatol. Venereol. 2011, 25, 74-81.

(2). Fabricant, D. S.; Farnsworth, N. R. The value of plants used in traditional medicine for drug discovery. Environ. Health. Perspect. 2001, 109, 69-75.

(3). Rauwald, H. W.; Brehm, O.; Odenthal, K. P. The involvement of a $\mathrm{Ca}^{2+}$ channel blocking mode of action in the pharmacology of Ammi visnaga fruits. Planta Med. 1994, 60, 101-105.

(4). Orr, T. S. Roger Altounyan: the man and his work. Respir. Med. 1989, 83, Suppl A, 3-6.

(5). (a) Conn, J. J.; Kissane, R. W.; Koons, R. A.; Clark, T. E. The treatment of angina pectoris with khellin. Ann. Intern. Med. 1952, 36, 1173-1178; (b) Osher, H. L.; Katz, K. H.; Wagner, D. J. Khellin in the treatment of angina pectoris. N. Engl. J. Med. 1951, 244, 315-321; (c) Dewar, H. A.; Grimson, T. A. Khellin in the treatment of angina of effort. Br. Heart J. 1950, 12, 54-60; (d) Anrep, G. V.; Barsoum, G. S.; et al. Ammi visnaga in the treatment of the anginal syndrome. Br. Heart J. 1946, 8, 171-177.

(6). (a) Deltour, G.; Binon, F.; Tondeur, R.; Goldenberg, C.; Henaux, F.; Sion, R.; Deray, E.; Charlier, R. Studies in the benzofuran series. VI. Coronary-dilating activity of alkylated and aminoalkylated derivatives of 3-benzoylbenzofuran. Arch. Int. Pharmacodyn. Ther. 1962, 139, 247-254; (b) Charlier, R.; Deltour, G.; Tondeur, R.; Binon, F. Studies in the benzofuran series. VII. Preliminary pharmacological study of 2-butyl-3-(3,5-diiodo-4-beta-Ndiethylaminoethoxybenzoyl)-benzofuran. Arch. Int. Pharmacodyn. Ther. 1962, 139, 255-264. 
(7). (a) Abdel-Fattah, A.; Aboul-Enein, M. N.; Wassel, G. M.; El-Menshawi, B. S. An approach to the treatment of vitiligo by khellin. Dermatologica 1982, 165, 136-140; (b) Vedaldi, D.; Caffieri, S.; Dall'Acqua, F.; Andreassi, L.; Bovalini, L.; Martelli, P. Khellin, a naturally occurring furochromone, used for the photochemotherapy of skin diseases: mechanism of action. Farmaco Sci. 1988, 43, 333-346; (c) Ashour, A.; El-Sharkawy, S.; Amer, M.; Abdel Bar, F.; Kondo, R.; Shimizu, K. Melanin biosynthesis inhibitory activity of compounds isolated from unused parts of Ammi visnaga. J. Cosmet. Dermatol. Sci. Appl. 2013, 30 (3B), 40-43.

(8). (a) Lee, J. K.; Jung, J. S.; Park, S. H.; Park, S. H.; Sim, Y. B.; Kim, S. M.; Ha, T. S.; Suh, H. W. Anti-inflammatory effect of visnagin in lipopolysaccharide-stimulated BV-2 microglial cells. Arch. Pharm. Res. 2010, 33, 1843-1850; (b) Abu-Hashem, A. A.; Youssef, M. M. Synthesis of new visnagen and khellin furochromone pyrimidine derivatives and their anti-inflammatory and analgesic activity. Molecules 2011, 16, 1956-1972.

(9). Elgazwy, A. S.; Edrees, M. M.; Ismail, N. S. Molecular modeling study bioactive natural product of khellin analogues as a novel potential pharmacophore of EGFR inhibitors. J. Enzyme Inhib. Med. Chem. 2013, 28, 1171-1181.

(10). Joshi, P.; Sonawane, V. R.; Williams, I. S.; McCann, G. J. P.; Gatchie, L.; Sharma, R.; Satti, N.; Chaudhuri, B.; Bharate, S. B. Identification of karanjin isolated from the Indian beech tree as a potent CYP1 enzyme inhibitor with cellular efficacy via screening of a natural product repository. MedChemComm 2018, 9, 371-382

(11). (a) Androutsopoulos, V. P.; Tsatsakis, A. M. Benzo[a]pyrene sensitizes MCF7 breast cancer cells to induction of G1 arrest by the natural flavonoid eupatorin-5-methyl ether, via activation of cell signaling proteins and CYP1-mediated metabolism. Toxicol. Lett. 2014, 230, 304-313; (b) Androutsopoulos, V.; Arroo, R. R.; Hall, J. F.; Surichan, S.; Potter, G. A. Antiproliferative and cytostatic effects of the natural product eupatorin on MDA-MB-468 human breast cancer cells due to CYP1-mediated metabolism. Breast Cancer Res. 2008, 10, R39; (c) Fukuda, I.; Nishiumi, 
S.; Mukai, R.; Yoshida, K.; Ashida, H. Catechins in tea suppress the activity of cytochrome P450 1A1 through the aryl hydrocarbon receptor activation pathway in rat livers. Int. J. Food Sci. Nutr. 2015, 66, 300-307; (d) Wu, Z.; Uchi, H.; Morino-Koga, S.; Nakamura-Satomura, A.; Kita, K.; Shi, W.; Furue, M. Z-Ligustilide inhibits benzo(a)pyrene-induced CYP1A1 upregulation in cultured human keratinocytes via ROS-dependent Nrf2 activation. Exp. Dermatol. 2014, 23, 260-265; (e) Hawas, U. W.; Gamal-Eldeen, A. M.; El-Toumy, S. A.; Meyer, J. J.; Hussein, A. A. Inhibition of the initiation stage of carcinogenesis by Salvia disermas constituents. Z. Naturforsch. C. 2009, 64, 831-839.

(12). (a) Horley, N. J.; Beresford, K. J. M.; Kaduskar, S.; Joshi, P.; McCann, G. J. P.; Ruparelia, K. C.; Williams, I. S.; Gatchie, L.; Sonawane, V. R.; Bharate, S. B.; Chaudhuri, B. (E)-3-(3,4,5Trimethoxyphenyl)-1-(pyridin-4-yl)prop-2-en-1-one, a heterocyclic chalcone is a potent and selective CYP1A1 inhibitor and cancer chemopreventive agent. Bioorg. Med. Chem. Lett. 2017, 27, 5409-5414; (b) Williams, I. S.; Joshi, P.; Gatchie, L.; Sharma, M.; Satti, N. K.; Vishwakarma, R. A.; Chaudhuri, B.; Bharate, S. B. Synthesis and biological evaluation of pyrrole-based chalcones as CYP1 enzyme inhibitors, for possible prevention of cancer and overcoming cisplatin resistance. Bioorg. Med. Chem. Lett. 2017, 27, 3683-3687; (c) Joshi, P.; McCann, G. J. P.; Sonawane, V. R.; Vishwakarma, R. A.; Chaudhuri, B.; Bharate, S. B. Identification of potent and selective CYP1A1 inhibitors via combined ligand and structurebased virtual screening and their in vitro validation in sacchrosomes and live human cells. $J$. Chem. Inf. Model. 2017, 57, 1309-1320.

(13). (a) Aziz, G. Michael condensation. Experiments with furochalcones and furoflavanones. Egypt. J. Chem. 1978, 19, 835-850; (b) Osman, S. A.; Yosef, H. A. A.; Hafez, T. S.; El-Sawy, A. A.; Mousa, H. A.; Ashraf, S.; Hassan, A. S. Synthesis and antibacterial activity of some novel chalcones, pyrazoline and 3-cyanopyridine derivatives based on khellinone as well as Ni (II), Co (II) and Zn (II) Complexes. Aust. J. Basic Appl. Sci. 2012, 6, 852-863. 
(14). (a) Shimizu, Y.; Nakatsuru, Y.; Ichinose, M.; Takahashi, Y.; Kume, H.; Mimura, J.; FujiiKuriyama, Y.; Ishikawa, T. Benzo[a]pyrene carcinogenicity is lost in mice lacking the aryl hydrocarbon receptor. Proc. Natl. Acad. Sci. U S A 2000, 97, 779-782; (b) Shimada, T.; FujiiKuriyama, Y. Metabolic activation of polycyclic aromatic hydrocarbons to carcinogens by cytochromes P450 1A1 and 1B1. Cancer Sci. 2004, 95, 1-6; (c) Shimada, T.; Inoue, K.; Suzuki, Y.; Kawai, T.; Azuma, E.; Nakajima, T.; Shindo, M.; Kurose, K.; Sugie, A.; Yamagishi, Y.; Fujii-Kuriyama, Y.; Hashimoto, M. Arylhydrocarbon receptor-dependent induction of liver and lung cytochromes P450 1A1, 1A2, and 1B1 by polycyclic aromatic hydrocarbons and polychlorinated biphenyls in genetically engineered C57BL/6J mice. Carcinogenesis 2002, 23, 1199-1207.

(15). Harvey, A. J.; Baell, J. B.; Toovey, N.; Homerick, D.; Wulff, H. A new class of blockers of the voltage-gated potassium channel Kv1.3 via modification of the 4- or 7-position of khellinone. $J$. Med. Chem. 2006, 49, 1433-1441.

(16). Baell, B. J.; Gable, W. R.; Harvey, J. A.; Toovey, N.; Herzog, T.; Hansel, W.; Wulff, H. Khellinone derivatives as blockers of the voltage-gated potassium channel Kv1.3: synthesis and immunosuppressive activity. J. Med. Chem. 2004, 47, 2326-2336.

(17). Horley, N. J.; Beresford, K. J.; Chawla, T.; McCann, G. J.; Ruparelia, K. C.; Gatchie, L.; Sonawane, V. R.; Williams, I. S.; Tan, H. L.; Joshi, P.; Bharate, S. S.; Kumar, V.; Bharate, S. B.; Chaudhuri, B. Discovery and characterization of novel CYP1B1 inhibitors based on heterocyclic chalcones: Overcoming cisplatin resistance in CYP1B1-overexpressing lines. Eur. J. Med. Chem. 2017, 129, 159-174.

(18). Walsh, A. A.; Szklarz, G. D.; Scott, E. E. Human cytochrome P450 1A1 structure and utility in understanding drug and xenobiotic metabolism. J. Biol. Chem. 2013, 288, 12932-12943.

(19). (a) Arlt, V. M.; Krais, A. M.; Godschalk, R. W.; Riffo-Vasquez, Y.; Mrizova, I.; Roufosse, C. A.; Corbin, C.; Shi, Q.; Frei, E.; Stiborova, M.; van Schooten, F.-J.; Phillips, D. H.; Spina, D. 
Pulmonary inflammation impacts on CYP1A1-mediated respiratory tract DNA damage induced by the carcinogenic air pollutant benzo[a]pyrene. Toxicol. Sci. 2015, 146, 213-225; (b) Rendic, S.; Guengerich, F. P. Contributions of human enzymes in carcinogen metabolism. Chem. Res. Toxicol. 2012, 25, 1316-1383.

(20). Endo, K.; Uno, S.; Seki, T.; Ariga, T.; Kusumi, Y.; Mitsumata, M.; Yamada, S.; Makishima, M. Inhibition of aryl hydrocarbon receptor transactivation and DNA adduct formation by CYP1 isoform-selective metabolic deactivation of benzo[a]pyrene. Toxicol. Appl. Pharmacol. 2008, 230, 135-143.

(21). Vichai, V.; Kirtikara, K. Sulforhodamine B colorimetric assay for cytotoxicity screening. Nat. Protoc. 2006, 1, 1112-1116.

(22). Hukkanen, J.; Lassila, A.; Paivarinta, K.; Valanne, S.; Sarpo, S.; Hakkola, J.; Pelkonen, O.; Raunio, H. Induction and regulation of xenobiotic-metabolizing cytochrome P450s in the human A549 lung adenocarcinoma cell line. Am. J. Respir. Cell Mol. Biol. 2000, 22, 360-366.

(23). Lin, P.; Chang, H.; Tsai, W. T.; Wu, M. H.; Liao, Y. S.; Chen, J. T.; Su, J. M. Overexpression of aryl hydrocarbon receptor in human lung carcinomas. Toxicol. Pathol. 2003, 31, 22-30.

(24). (a) Moorthy, B.; Chu, C.; Carlin, D. J. Polycyclic aromatic hydrocarbons: from metabolism to lung cancer. Toxicol. Sci. 2015, 145, 5-15; (b) Androutsopoulos, V. P.; Tsatsakis, A. M.; Spandidos, D. A. Cytochrome P450 CYP1A1: wider roles in cancer progression and prevention. BMC Cancer 2009, 9, 187.

(25). Hassan, M. A.; Zubair, M. U. Khellin. In Analytical Profiles of Drug Substances, Klaus, F., Ed. Academic Press: 1981; Vol. 9, pp 371-396.

(26). Baell, J. B.; Wulff, H.; Chandy, G. K.; Norton, R. S. Novel chalcone derivatives and uses thereof. WO2003076407A1, September 18, 2003. 
(27). El-Sawy, E. R.; Ebaid, M. S.; Abo-Salem, H. M.; El-Hallouty, S.; Kassem, E. M.; Mandou, A. H. Synthesis and biological activity of novel series of 4-methoxy, and 4,9-dimethoxy-5substituted furo[2,3-g]-1,2,3-benzoxathiazine-7,7-dioxide derivatives. J. Adv. Res. 2014, 5, 337346.

(28). (a) Mahale, S.; Bharate, S. B.; Manda, S.; Joshi, P.; Bharate, S. S.; Jenkins, P. R.; Vishwakarma, R. A.; Chaudhuri, B. Biphenyl-4-carboxylic acid [2-(1H-indol-3-yl)-ethyl]-methylamide (CA224), a nonplanar analogue of fascaplysin, inhibits Cdk4 and tubulin polymerization: evaluation of in vitro and in vivo anticancer activity. J. Med. Chem. 2014, 57, 9658-9672; (b) Mahale, S.; Bharate, S. B.; Manda, S.; Joshi, P.; Jenkins, P. R.; Vishwakarma, R. A.; Chaudhuri, B. Antitumour potential of BPT: a dual inhibitor of cdk4 and tubulin polymerization. Cell Death Dis. 2015, 6, e1743. 


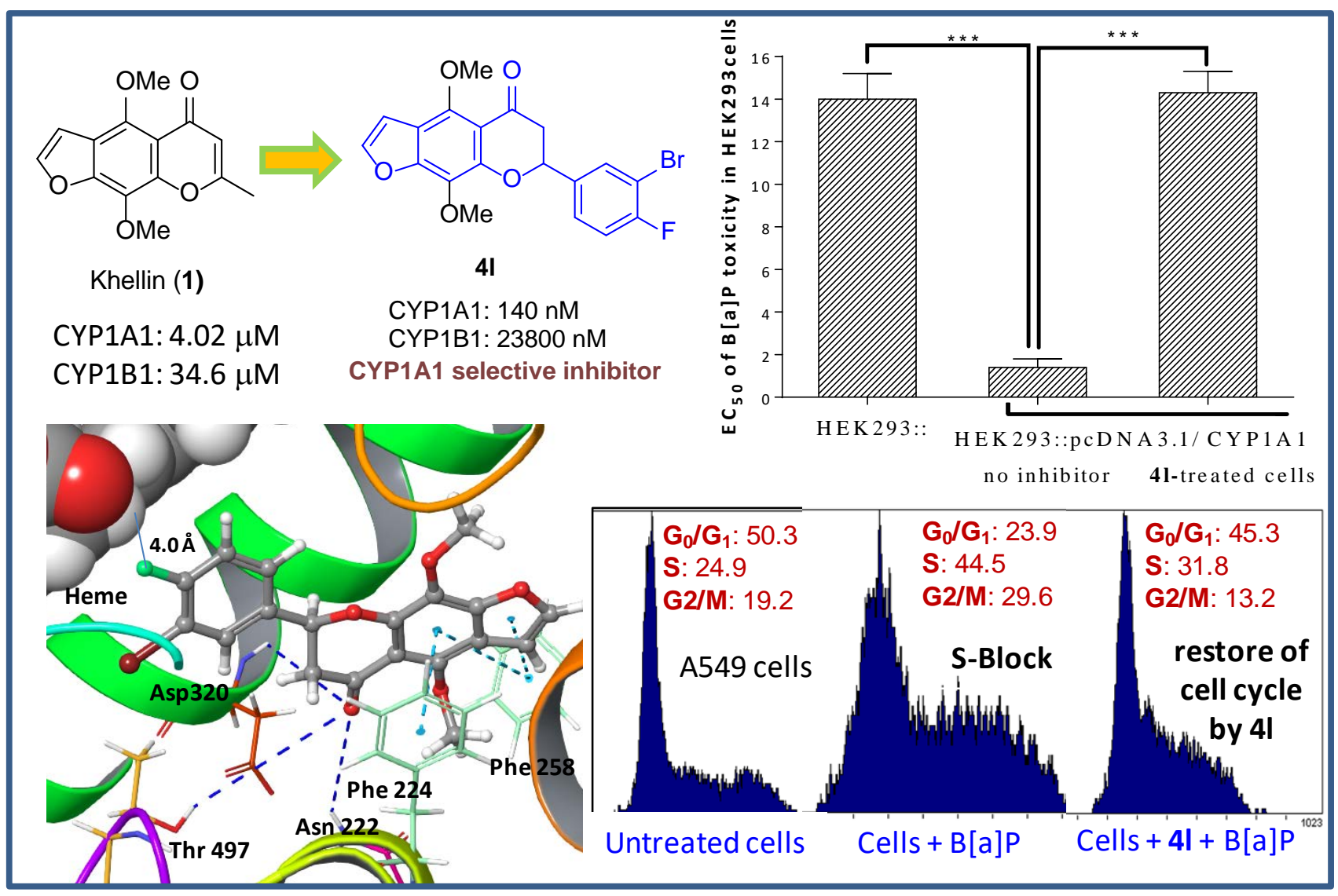

